\title{
HTLV-1 viral oncogene HBZ drives bone destruction in adult T cell leukemia
}

\author{
Jingyu Xiang, ${ }^{1}$ Daniel A. Rauch,, Devra D. Huey, ${ }^{2}$ Amanda R. Panfil, ${ }^{2}$ Xiaogang Cheng, ${ }^{1}$ \\ Alison K. Esser, ${ }^{1}$ Xinming Su, ${ }^{1}$ John C. Harding, ${ }^{1}$ Yalin Xu, ${ }^{1}$ Gregory C. Fox, ${ }^{1}$ Francesca Fontana, \\ Takayuki Kobayashi, ${ }^{1}$ Junyi Su, ${ }^{1}$ Hemalatha Sundaramoorthi,, Wing Hing Wong, ${ }^{1}$ Yizhen Jia, ${ }^{1}$ \\ Thomas J. Rosol, ${ }^{2,3}$ Deborah J. Veis, ${ }^{4}$ Patrick L. Green, ${ }^{2}$ Stefan Niewiesk, ${ }^{2}$ Lee Ratner, ${ }^{1}$ \\ and Katherine N. Weilbaecher ${ }^{1}$ \\ 'Department of Medicine, Division of Oncology, Washington University School of Medicine, St. Louis, Missouri, USA. \\ ${ }^{2}$ Center for Retrovirus Research, Department of Veterinary Biosciences, The Ohio State University, Columbus, Ohio, USA. \\ ${ }^{3}$ Department of Biomedical Sciences, Heritage College of Osteopathic Medicine, Ohio University, Athens, Ohio, USA. \\ ${ }^{4}$ Department of Medicine, Division of Bone and Mineral Diseases, St. Louis, Missouri, USA.
}

Osteolytic bone lesions and hypercalcemia are common, serious complications in adult $\mathrm{T}$ cell leukemia/lymphoma (ATL), an aggressive T cell malignancy associated with human T cell leukemia virus type 1 (HTLV-1) infection. The HTLV-1 viral oncogene HBZ has been implicated in ATL tumorigenesis and bone loss. In this study, we evaluated the role of HBZ on ATL-associated bone destruction using HTLV-1 infection and disease progression mouse models. Humanized mice infected with HTLV-1 developed lymphoproliferative disease and continuous, progressive osteolytic bone lesions. HTLV-1 lacking HBZ displayed only modest delays to lymphoproliferative disease but significantly decreased disease-associated bone loss compared with HTLV-1-infected mice. Gene expression array of acute ATL patient samples demonstrated increased expression of RANKL, a critical regulator of osteoclasts. We found that HBZ regulated RANKL in a c-Fos-dependent manner. Treatment of HTLV-1-infected humanized mice with denosumab, a monoclonal antibody against human RANKL, alleviated bone loss. Using patient-derived xenografts from primary human ATL cells to induce lymphoproliferative disease, we also observed profound tumor-induced bone destruction and increased $c$-Fos and RANKL gene expression. Together, these data show the critical role of HBZ in driving ATL-associated bone loss through RANKL and identify denosumab as a potential treatment to prevent bone complications in ATL patients.

Authorship note: JX, DAR, and DDH contributed equally.

Conflict of interest: The authors have declared that no conflict of interest exists.

Copyright: (c) 2019, American Society for Clinical Investigation.

Submitted: March 13, 2019

Accepted: September 4, 2019

Published: October 3, 2019

Reference information: JCI Insight. 2019;4(19):e128713.

https://doi.org/10.1172/jci.

insight.128713.

\section{Introduction}

Human T cell leukemia virus type 1 (HTLV-1) is a retrovirus estimated to infect 5-10 million people worldwide and causes an aggressive CD4 T cell malignancy called adult T cell leukemia/lymphoma (ATL) (1). Like HIV-1, HTLV-1 is lymphotropic and mainly infects CD4+ T cells (2). About $2 \%-5 \%$ of HTLV-1infected people develop ATL over the course of their lifetime. Reminiscent of multiple myeloma, the most common complication in ATL patients is severe osteolytic bone destruction associated with hypercalcemia and fractures, especially in patients with acute and lymphomatous ATL subtypes $(3,4)$.

There are 2 oncogenes encoded in the HTLV-1 genome: Tax and HBZ (HTLV-1 bZIP factor) (5). Tax is encoded by the plus strand of the proviral genome and is involved in transformation, viral transcription, and cell cycle regulation $(6,7)$. Expression of Tax alone is sufficient to drive the rapid onset of lymphoma/ leukemia in animal models (8-10). Additionally, using transgenic mice expressing Tax under the granzyme B promoter (Gzmb-Tax), we have previously shown that $\mathrm{Tax}^{+}$tumor cells produce osteoclast-activating factors, including IL-6, MCSF, IL- $1 \alpha$ and IL-1 $\beta$, TNF- $\alpha$, and TGF- $\beta$, indicating that Tax may play a role in the development of bone loss in this model (8). Despite the importance of Tax in the development and progression of ATL, only $30 \%-40 \%$ of ATL patients have detectable Tax expression (11). This is likely because Tax is immunogenic and targeted by cytotoxic T cells. Thus, in order to evade immune surveillance, the Tax gene is usually deleted or silenced in ATL samples (12-14). The low Tax expression in the majority of the ATL patients suggests that there are other viral factors responsible for bone disease. HBZ is constitutively expressed in all ATL patients and is also involved in the development and progression of ATL (15). HBZ is involved in viral 
transcription, proliferation, infectivity, and persistence (16). Interestingly, $H B Z$ RNA itself promotes cell proliferation $(17,18)$. We recently demonstrated that Gzmb-HBZ transgenic mice develop lymphoproliferative disease (LPD), splenomegaly, and osteolytic disease after an 18-month latency. These mice also exhibit elevated levels of inflammatory and bone-activating factors such as IL-6, IL-3, and MCP-1 (19), indicating that HBZ may also contribute to ATL-associated bone pathology. Despite these findings, the precise mechanism for how HBZ contributes to lymphoproliferative and bone disease in the context of ATL remains unclear.

The bone matrix undergoes dynamic changes controlled by the balance between bone-forming osteoblasts and bone-resorbing osteoclasts, both of which are regulated by signals from bone-resident osteocytes $(20,21)$. In pathological conditions such as inflammation or cancer (e.g., breast cancer, multiple myeloma), this balance is often biased in favor of bone loss $(22,23)$. Osteoblast-derived cytokines such as macrophage $\mathrm{CSF}(\mathrm{MCSF})$ and receptor activator of NF- $\mathrm{kB}$ ligand (RANKL) are key factors required for osteoclast differentiation. Immune cells such as $\mathrm{T}$ cells and $\mathrm{B}$ cells can also produce MCSF and RANKL, enhancing osteoclastogenesis (24). Indeed, ATL patients with hypercalcemia were found to have elevated $R A N K L$ expression (25). In line with this finding, in vitro coculture of BM macrophages with an HTLV-1-infected T cell line induced significantly more osteoclast formation compared with coculture with a normal $\mathrm{T}$ cell line (26). In addition to RANKL, bone-acting factors such as parathyroid hormone-related protein (PTHrP), MIP-1 $\alpha$, MCP-1, and IL-6 are also important mediators of malignant hypercalcemia and osteolytic bone disease. We have shown that PTHrP, its receptor PTH1R, and MIP-1 $\alpha$ are markedly upregulated during HTLV-1 immortalization of primary peripheral blood mononuclear cells (PBMCs) (27), suggesting that multiple factors may directly or indirectly affect bone in ATL.

Humanized mouse models have been widely used to study various types of diseases such as human infectious disease and cancer $(28,29)$. To evaluate the importance of HBZ on ATL-associated bone disease, we utilized CD34+ humanized mice infected with HTLV-1 and HTLV-1 $\triangle$ HBZ, which lacks the HBZ protein. In this study, we found that HTLV-1-infected humanized mice developed LPD, as well as progressive osteolytic bone disease, with increased osteoclast surface and number that correlated with LPD burden. We found that HTLV-1 $\triangle$ HBZ-infected humanized mice developed LPD with a slight delay to disease but had no disease-associated bone loss. Further, we found that HBZ regulated RANKL expression through a c-Fos-dependent mechanism. Selective blockade of human RANKL with denosumab decreased disease-associated bone loss in HTLV-1-infected humanized mice. Finally, we evaluated bone loss in primary ATL patient-derived xenografts and found that mice inoculated with human ATL cells developed splenomegaly and significant bone loss and had increased RANKL and FOS gene expression. Taken together, these data suggest that HBZ is a key driver of tumor-associated bone disease in ATL.

\section{Results}

HTLV-1-infected humanized mice develop LPD and splenomegaly. We employed HTLV-1 inoculation of humanized mice because it recapitulates all stages of HTLV-1 pathogenesis from infection to the development of LPD. In this animal model, a human hematopoietic system is established in NOD-scid-IL2R $\gamma^{-1}$ (NSG) mice using human $\mathrm{CD}_{3} 4^{+}\left(\mathrm{hCD} 34^{+}\right)$umbilical cord blood, as previously described $(30,31)$, to produce humanized mice. After the establishment of human hematopoietic cells, mice were infected with HTLV-1 and then monitored for disease development. We found that HTLV-1 humanized mice developed lymphocytic transformation by 3 weeks and progressed to end-stage LPD by 6-8 weeks (median survival, 5-6 weeks) (Figure 1, A and $\mathrm{B})$, as we previously published $(31,32)$. Disease progression was evaluated by flow cytometry of PBMC (Supplemental Figure 1; supplemental material available online with this article; https://doi.org/10.1172/ jci.insight.128713DS1). In age-matched, uninfected humanized mice, the percentage of hCD4 ${ }^{+} \mathrm{T}$ cells was maintained at around 3\%-5\%. After HTLV-1 infection, however, humanized mice developed splenomegaly and a rapid increase in $\mathrm{hCD} 4^{+} \mathrm{T}$ cells in the peripheral blood (Figure 1, C-E). The clonality of HTLV-1infected $\mathrm{T}$ cells in our humanized mouse model was assessed using $\mathrm{T}$ cell receptor rearrangement analysis. The LPD in our HTLV-1-infected humanized mouse was oligoclonal (Supplemental Table 1), which was consistent with findings from other groups $(30,33)$. To evaluate the role of HBZ in LPD and bone loss in the humanized mouse model, age-matched mice were infected with HTLV-1 $\triangle$ HBZ. HTLV-1 $\triangle$ HBZ was generated by introducing a $\mathrm{G}$ to $\mathrm{A}$ stop codon point mutation that causes the termination of the $\mathrm{HBZ}$ reading frame at amino acid 8, resulting in lack of HBZ protein but intact HBZ RNA (16). HTLV-1 $\triangle H B Z$-infected mice developed LPD and splenomegaly. We observed a 7-10 day delay in the development of fulminant LPD in the HTLV-1 $\triangle$ HBZ-infected animals but no change in disease mortality (Figure 1A) and mouse total body 
A Survival of humanized mice post HTLV-1 infection

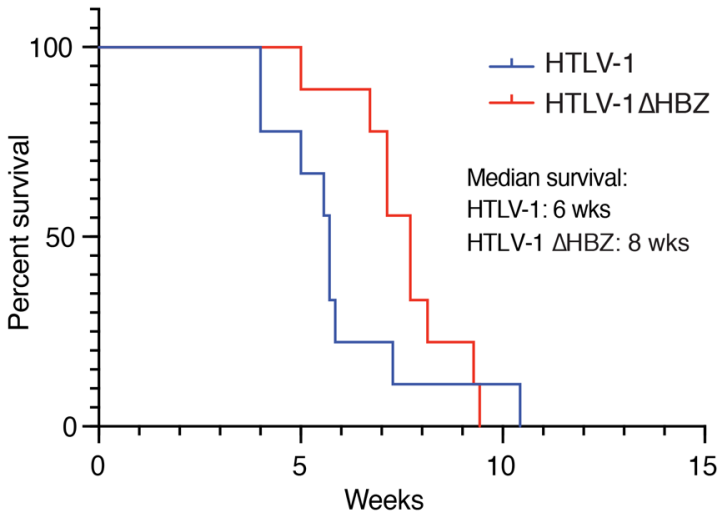

C

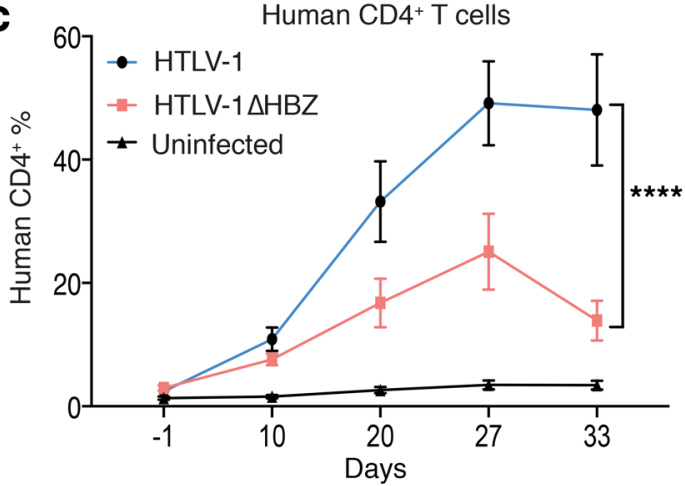

$\mathbf{E}$

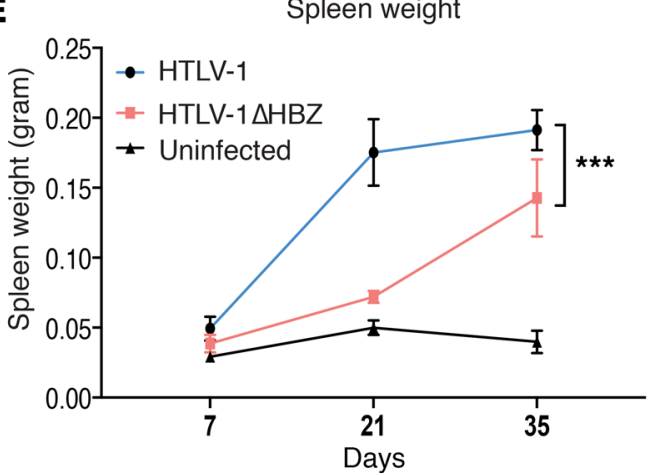

B

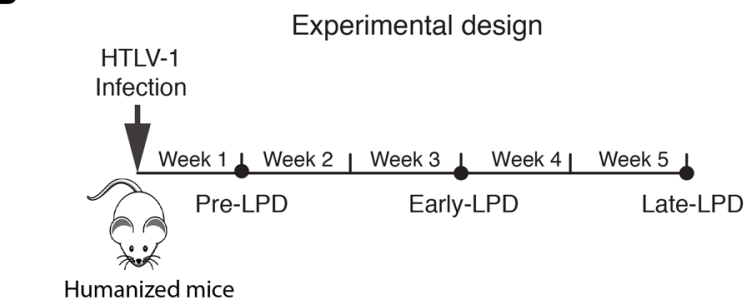

D

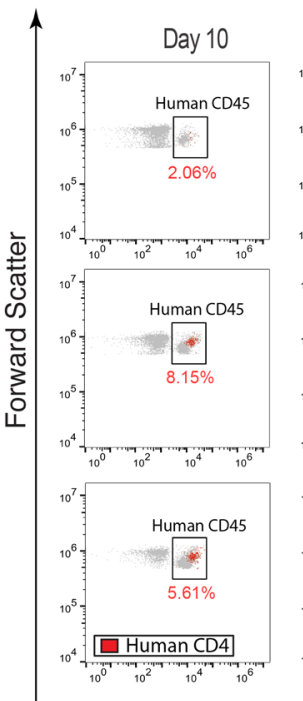

Days after infection
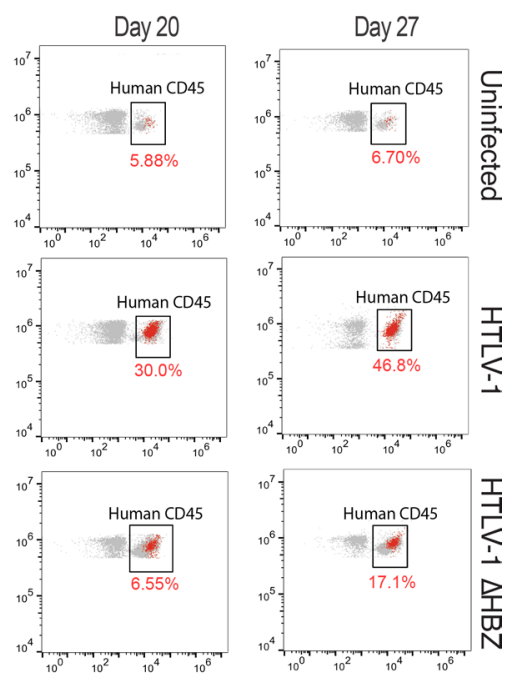

Human CD45

Figure 1. HTLV-1-infected humanized mice develop lymphoproliferative disease (LPD). (A) Kaplan-Meier survival curves of humanized mice infected with HTLV-1 $(n=9)$ and HTLV-1 $\triangle$ HBZ $(n=9)$ viruses. (B) Experimental design: humanized mice were infected with HTLV-1 or HTLV-1 $\Delta$ HBZ and examined at pre-LPD (1 week after infection), early-LPD (3 weeks after infection), and late-LPD (5 weeks after infection). (C) The average percentage of human CD4 ${ }^{+} T$ cells present in the peripheral blood of humanized mice infected with HTLV-1 $(n=8)$ and HTLV-1 $\Delta$ HBZ $(n=8)$ viruses obtained by flow cytometry over the course of 5 weeks. Error bars represent \pm SEM and ${ }^{* * *} P<0.0001$ (2-way ANOVA). (D) Representative scatter plots of flow data obtained on days 10,20 , and 27 depicting the expansion of human $\mathrm{CD} 5^{+}$(box) $\mathrm{CD}_{4}^{+}$(red dots) T cells in peripheral blood of infected humanized mice over the course of disease progression. (E) The average spleen weight as an additional measure of LPD in infected humanized mice, obtained on days 7, 21, and 35 . Error bars represent \pm SEM. ${ }^{* *} P<0.001(2$-way ANOVA).

weight (Supplemental Figure 2A). Proviral load measured by Tax copy number per cell also trended down in HTLV-1 $\triangle$ HBZ-infected animals compared with the WT-infected mice but was not significantly different between the 2 groups (Supplemental Figure 2B). These data suggest that HBZ contributes but is not required for the development of the LPD after HTLV-1 infection.

HTLV-1-infected humanized mice develop disease-associated bone loss. Osteolytic bone disease is a common complication of ATL in patients. We therefore evaluated bones from HTLV-1-infected humanized mice at various time points after HTLV-1 infection and transformation by microcomputed tomography $(\mu \mathrm{CT})$ anal- 
A

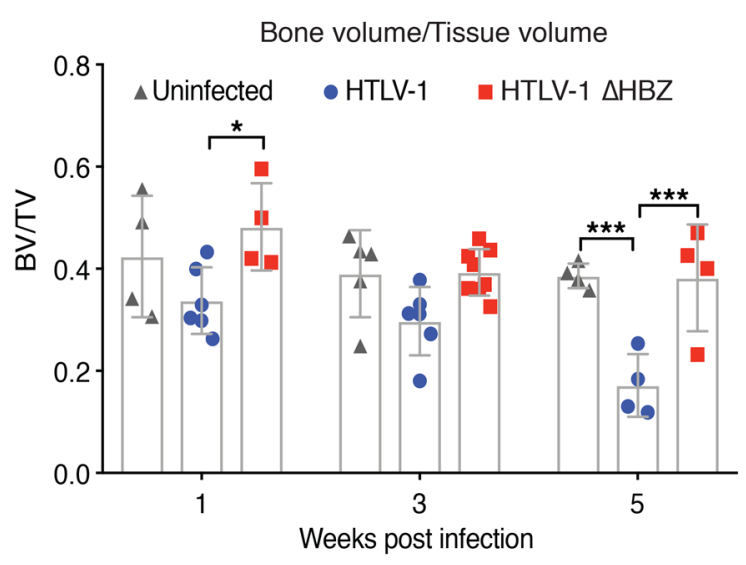

B

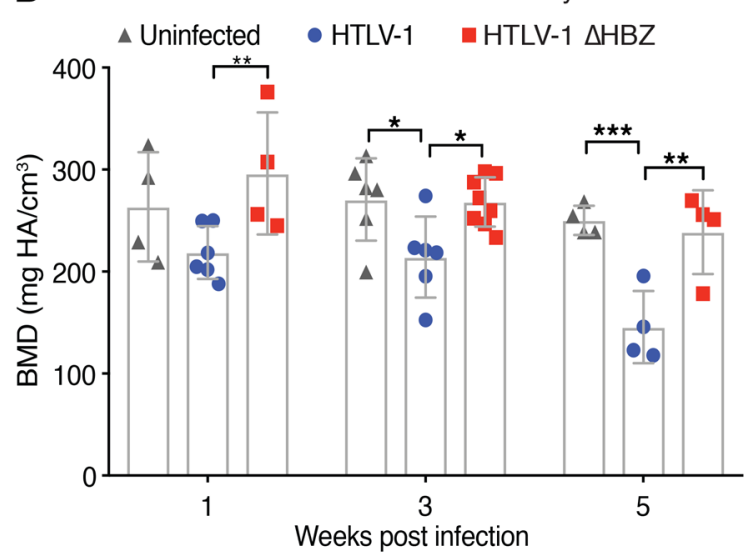

C

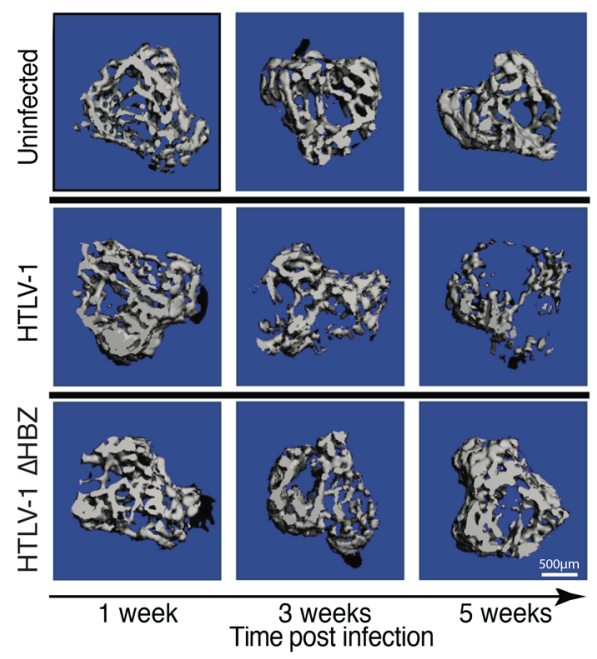

D

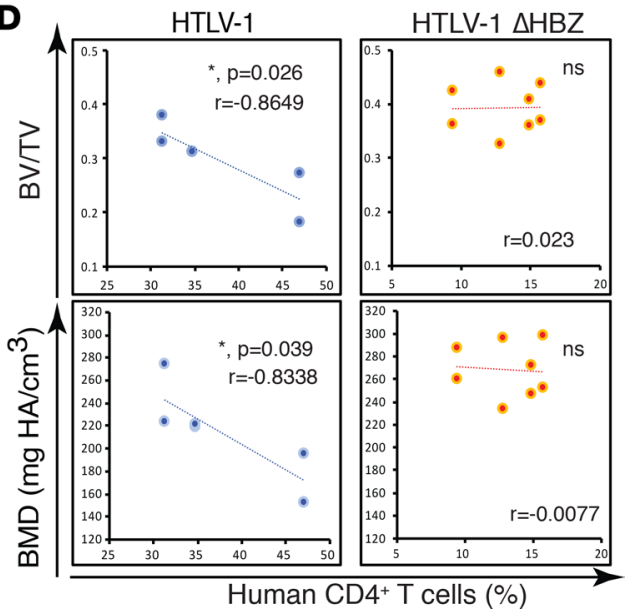

Figure 2. Deletion of HBZ partially rescues tumor-associated bone loss in HTLV-1-infected humanized mice. (A and B) $\mu C T$ analysis for calculation of trabecular bone to tissue volume ratio (BV/TV) and bone mineral density (BMD) in humanized mice infected with HTLV-1 and HTLV-1 $\triangle H B Z$ viruses 1 week, 3 weeks, and 5 weeks after infection ( $n=4-6$ bones per group). Error bars represent \pm SEM. ${ }^{*} P<0.05 ;{ }^{* *} P<0.01$; ${ }^{* * *} P<0.001$ ( 2 -way ANOVA). (C) Representative 3-D reconstruction images of tibial trabecular bone in humanized mice infected with HTLV-1 and HTLV-1 $\triangle$ HBZ viruses 1,3 , and 5 weeks after infection. (D) Scatter plots of the correlation (as indicated by linear best fit curve) between trabecular bone density (as measured by either BV/TV or $B M D$ ) on the $y$ axis vs. percentage of human CD4+ T cells in the peripheral blood of humanized mice infected with HTLV-1 and HTLV-1 $\triangle$ HBZ 3 weeks after infection ( $n=6-8$ bones per group). $R$ value and $P$ value (Pearson correlation coefficient test was adopted to determine statistically significant correlation between the 2 groups) is shown; ns, $P>0.05$.

ysis and histologic examination. We found that as early as 3 weeks after infection, HTLV-1-infected humanized mice had significant trabecular bone loss, as measured by both bone volume/tissue volume (BV/TV) and bone mineral density (BMD) (Figure 2, A-C). These bone parameters worsened with time and were negatively correlated with disease burden ( $\mathrm{CD} 4^{+} \mathrm{T}$ cells in peripheral blood) in HTLV-1-infected mice (Figure 2D). Mice infected with HTLV-1 $\triangle \mathrm{HBZ}$, by contrast, exhibited no evidence of significant bone loss compared with uninfected controls despite evidence of ongoing LPD. Likewise, we found no significant correlation of bone parameters with $\mathrm{CD} 4^{+} \mathrm{T}$ cell numbers in HTLV-1 $\triangle \mathrm{HBZ}$-infected mice. Bone histomorphometric assessments of osteoclast number by tartrate-resistant acid phosphatase (TRAP) staining revealed that HTLV1-infected mice had time-dependent increases in osteoclast number (Oc.\#/BS) and surface (Oc.S/BS). In HTLV-1 $\triangle$ HBZ-infected mice, however, we found no change in osteoclast numbers, despite LPD infiltration in BM (Figure 3, A-C). Osteoclast number and surface positively correlated with disease burden $\left(\mathrm{CD} 4^{+} \mathrm{T}\right.$ cells in peripheral blood) in HTLV-1, but not in HTLV-1 $\triangle$ HBZ-infected mice (Figure 3, D and E). Together, these data support a role for HBZ in disease-associated bone loss using a humanized mouse model.

$H B Z$ regulates the expression of the osteoclastogenic factor RANKL through $c$-Fos. Having observed that deletion of HBZ protein $(\triangle \mathrm{HBZ})$ was sufficient to rescue the ATL-associated increase in osteoclast number and 
A
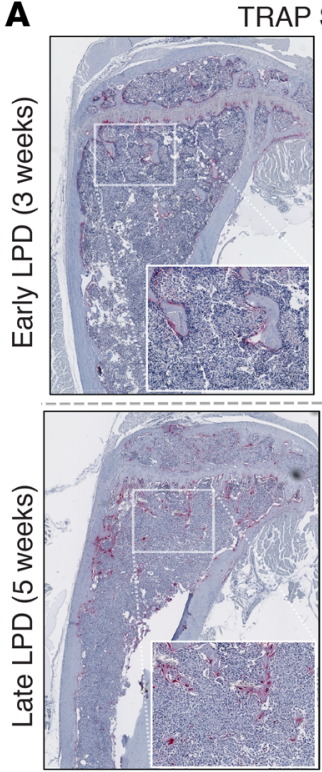

HTLV-1

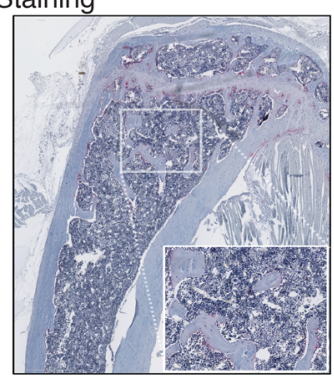

B
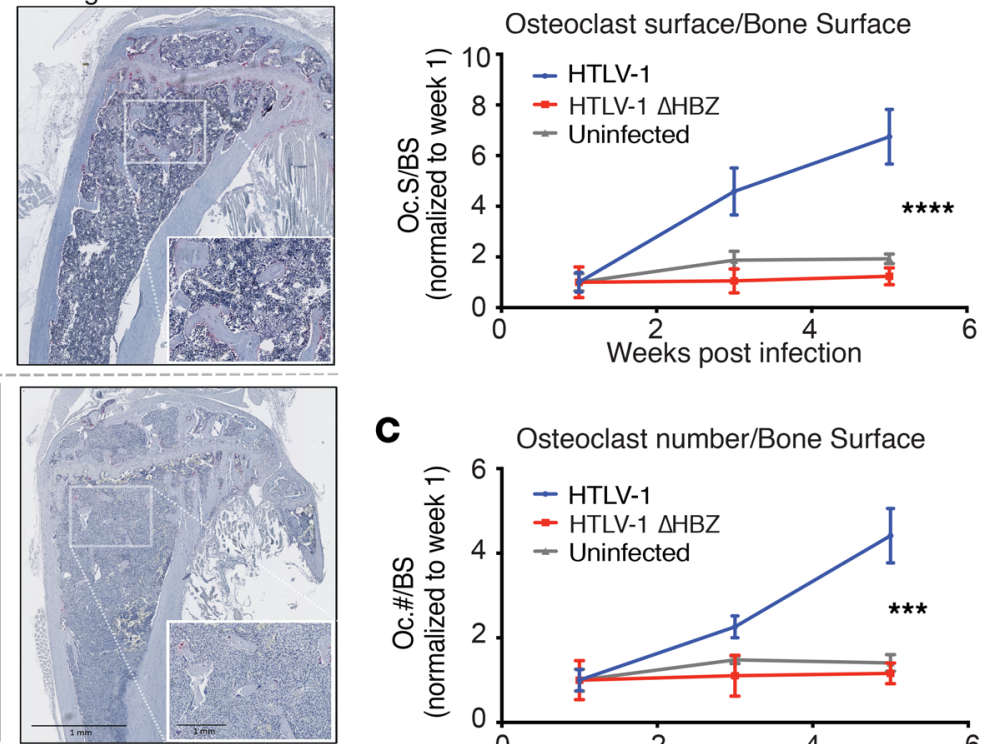

HTLV-1 $\triangle H B Z$

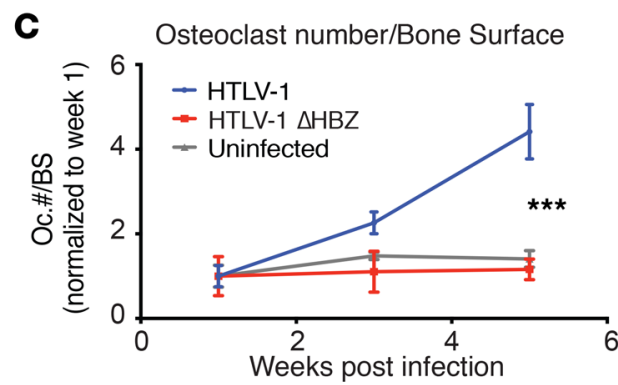

$\mathbf{E}$

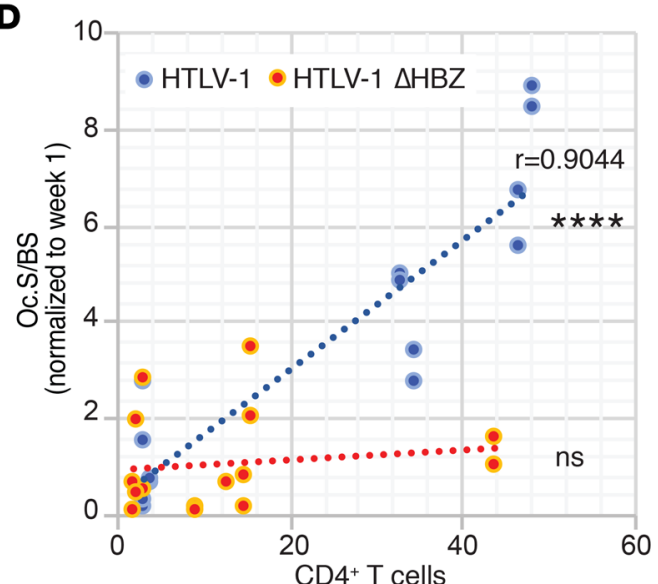

Figure 3. HTLV-1-infected humanized mice had increased osteoclast number in vivo. (A) Histomorphometry of formalin-fixed, paraffin-embedded, decalcified tibia from humanized mice infected with HTLV-1 and HTLV-1 $\triangle$ HBZ virus showing tartrate resistant acid phosphatase (TRAP) staining at early-LPD ( 3 weeks after infection) and lateLPD ( 5 weeks after infection). Scale bars: $1 \mathrm{~mm}$ on both $10 \times$ magnification and $20 \times$ (inset) magnification. (B and C) Quantification of osteoclast surface per bone surface and osteoclast number per bone surface normalized to week 1 ( $n>3$ bones per group). Error bars represent \pm SEM. ${ }^{* * *} P<0.001 ;{ }^{* * *} P<0.0001$ (2-way ANOVA comparing WT with $\triangle \mathrm{HBZ}$ ). ( $\mathbf{D}$ and $\mathbf{E})$ Scatter plots of the correlation (as indicated by linear best fit curve) between osteoclast surface/ bone surface or osteoclast number/bone surface on the $y$ axis vs. percentage of human CD4+ $\mathrm{T}$ cells in the peripheral blood. $P$ value (Pearson correlation coefficient test was adopted to determine statistically significant correlation between the 2 groups) is shown; ns, $P>0.05$.

bone loss, we next asked how HBZ could regulate osteoclastogenesis. RANKL is a critical factor regulating the development and function of bone-resorbing osteoclasts (20). It has been previously reported in a small cohort of patients that RANKL is highly expressed in ATL cells from patients with hypercalcemia, compared with ATL cells from patients without hypercalcemia (25). We recapitulated this finding in an analysis of the published microarray gene expression data (34) from acute ATL patient PBMCs $(n=26)$ vs. CD4 ${ }^{+}$ $\mathrm{T}$ cells from healthy donors $(n=21)$ and found that $R A N K L$ gene expression was significantly elevated in ATL patients compared with healthy patients (Figure $4 \mathrm{~A}$ ).

These data from human patients are consistent with our previous finding of elevated $R A N K L$ expression in murine $\mathrm{CD}^{+} \mathrm{T}$ lymphocytes from Gzmb-HBZ transgenic mice (in which $\mathrm{T}$ cell activation drives HBZ 
A

TNFSF11 (RANKL) Gene Expression (Acute ATL vs Healthy Donor)
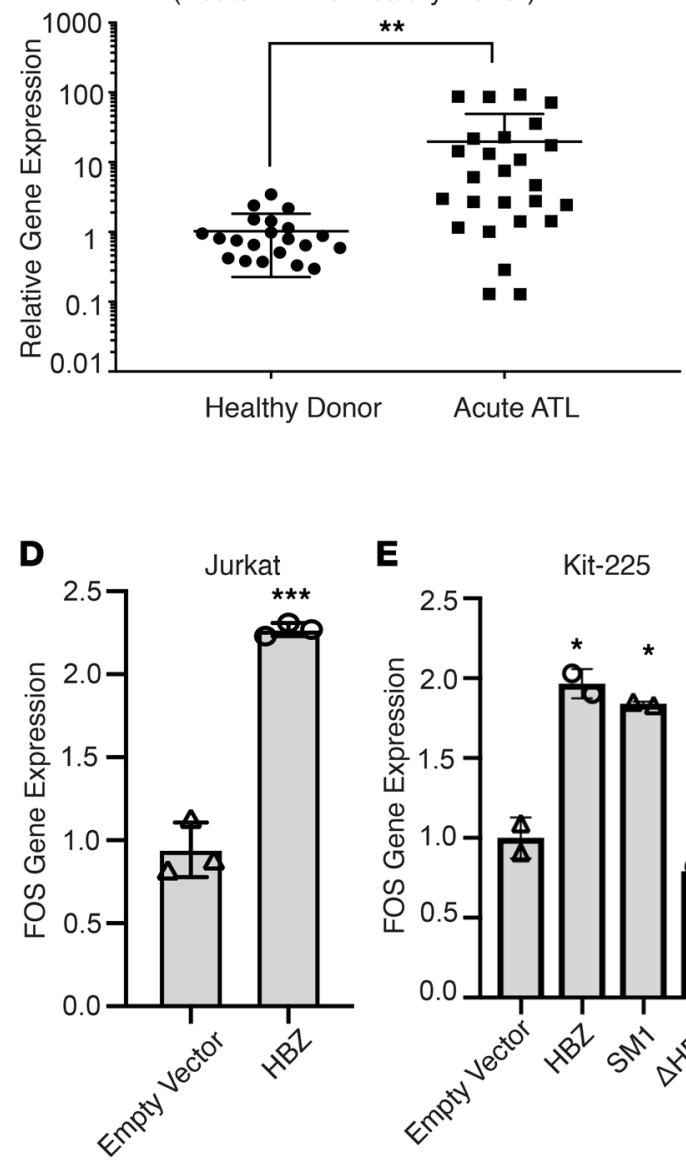

E
B
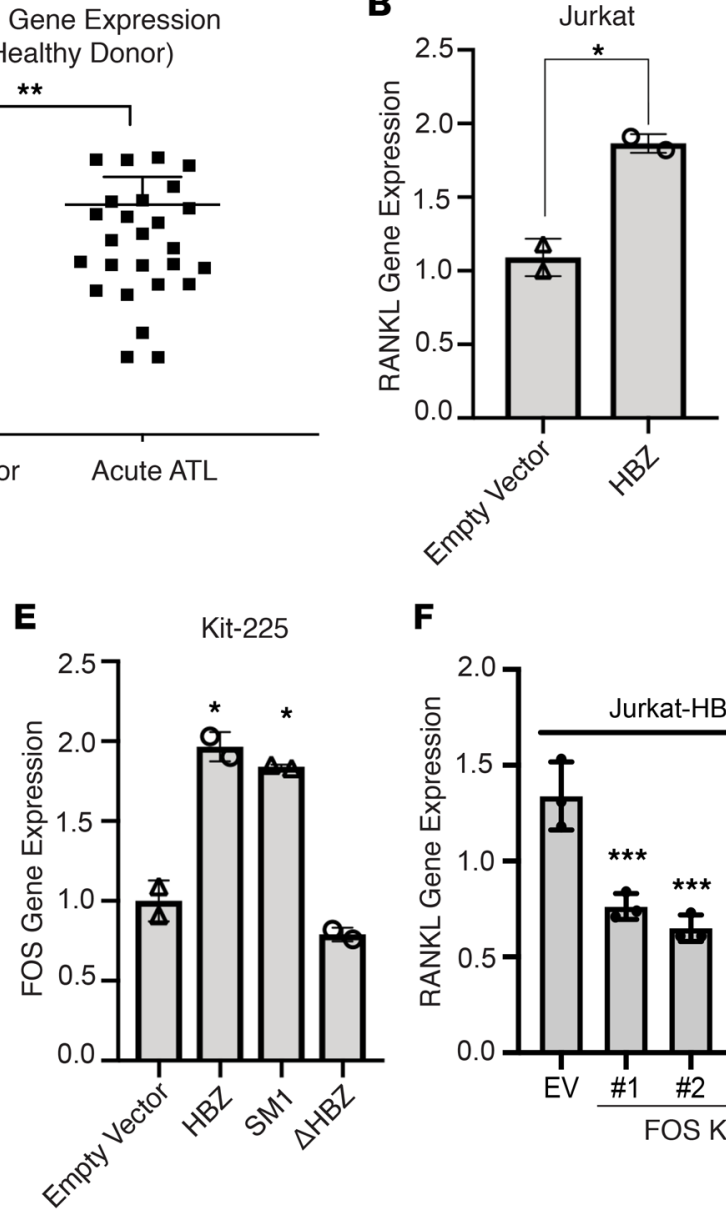

$\mathbf{F}$
C
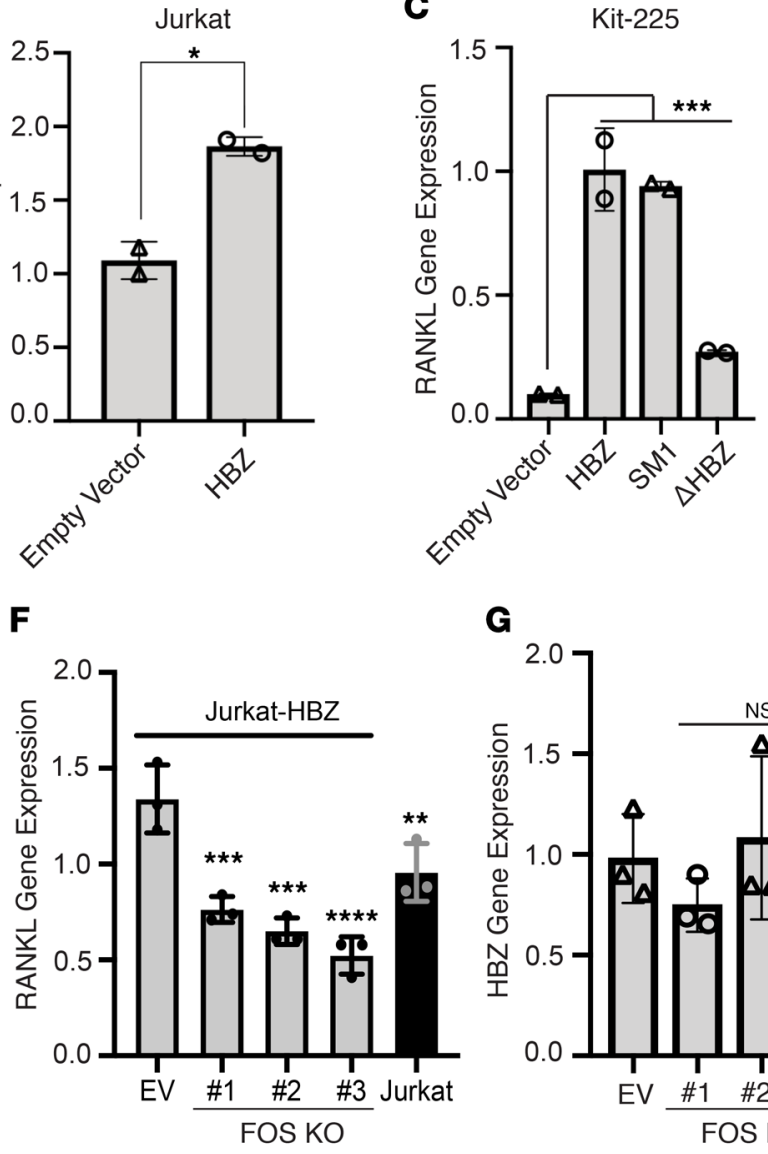

G

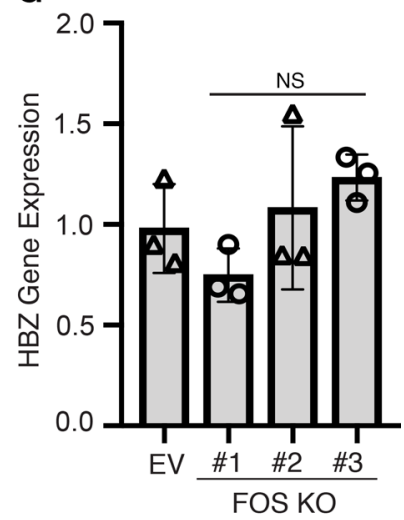

Figure 4. HBZ regulates the expression of the osteoclastogenic factor RANKL through c-Fos. (A) Gene expression data obtained from Gene Expression Omnibus at the NCBI (CSE33615). RNA from PBMCs of 26 patients with acute ATL vs. 21 normal uninfected HTLV-1-negative, healthy donors, normalized to actin shown as log fold over average TNFSF11 (RANKL) gene expression in normal subjects. (B) Quantitative PCR (qPCR) detection of RANKL transcription in Jurkat cells compared with HBZ-expressing Jurkat cells. (C) qPCR detection of RANKL transcription in Kit-225 cells expressing WT HBZ and 2 HBZ mutants; HBZ (SM1) encodes WT HBZ protein but carries silent mutations that disrupt HBZ RNA sequence and secondary structure; HBZ ( $\triangle \mathrm{HBZ}$ ) expressed WT HBZ RNA but does not express HBZ protein. (D) qPCR detection of FOS transcription in Jurkat cells compared with HBZ-expressing Jurkat cells. (E) qPCR detection of FOS transcription in Kit-225 cells expressing WT HBZ and 2 HBZ mutants: SM1 and $\triangle \mathrm{HBZ}$. (F) qPCR detection of RANKL (G) and HBZ transcription in HBZ-expressing Jurkat cells (Jurkat-HBZ) in the presence and absence of FOS. Data is representative of 2-3 biological replicates. Error bars in this figure represent \pm SEM. ${ }^{*} P<0.05$; ${ }^{* *} P<0.01$; ${ }^{* *} P<0.001$ (2-tailed distribution, homoscedastic student's $t$ test for 2 groups or 1-way ANOVA for multiple comparison).

expression) (19). To confirm these results, we used the inducible Tet-on Tax1 expression in Jurkat cells (35). In this system, we did not observe changes in RANKL gene expression after induction of Tax expression (Supplemental Figure 3, A and B). The observation that Tax did not induce RANKL prompted us to test whether HBZ induces upregulation of RANKL in 2 human lymphoid cell lines, Jurkat and Kit-225. We overexpressed HBZ in the human Jurkat $\mathrm{T}$ cell line and observed upregulation of $R A N K L$ gene expression by quantitative PCR (qPCR) (Figure 4B). HBZ protein and RNA have been shown to have distinct functions. HBZ protein has been shown to regulate genes associated with $\mathrm{T}$ cell immunological processes, while HBZ RNA has been associated with genes involved in cell cycle, proliferation, and survival $(17,18)$. To determine the specific contribution of HBZ protein and RNA to the increased RANKL gene expression, we expressed various HBZ mutants (WT, $\triangle \mathrm{HBZ}, \mathrm{SM1}$ ) in the human $\mathrm{T}$ cell line Kit-225. The $\triangle \mathrm{HBZ}$ mutant expresses no HBZ protein; however, the secondary structure of the HBZ RNA is intact (16). The HBZ SM1 mutant has a silent mutation in the third nucleotide position of the first 210 amino acids. This construct alters the RNA secondary structure but encodes the WT HBZ protein (HBZ protein only) (17). Using these various HBZ mutants, we found that the upregulation of RANKL gene expression in Kit-225 cells was mainly contributed by HBZ protein (Figure 4C).

To investigate whether $\mathrm{HBZ}$ regulates $R A N K L$ expression directly or indirectly through another transcription factor, we turned to a publicly available HBZ-FLAG ChIP sequencing (ChIP-seq) dataset (36). In 
A Experimental design

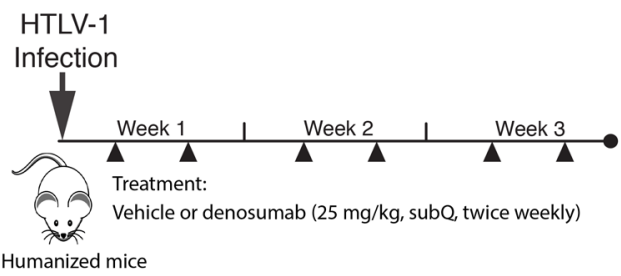

Humanized mice

B Bone volume/Tissue volume

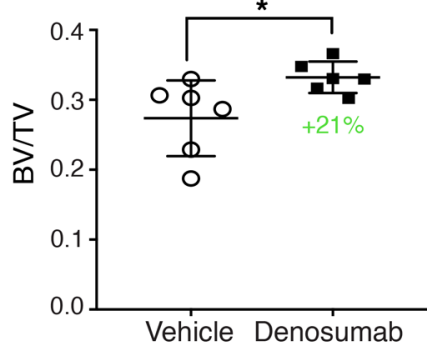

C
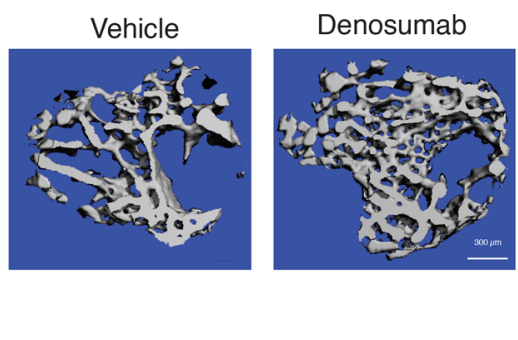

D Bone mineral density

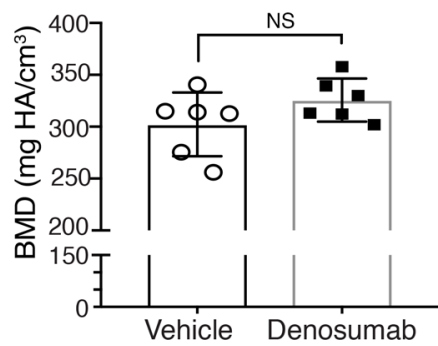

E

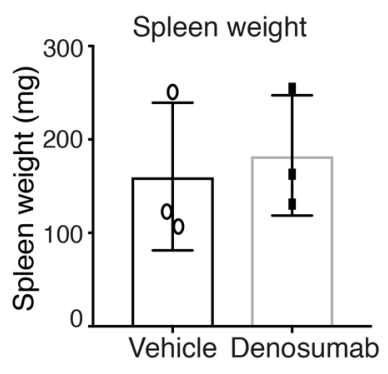

F

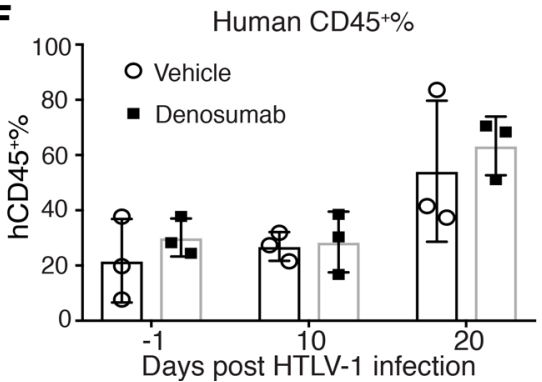

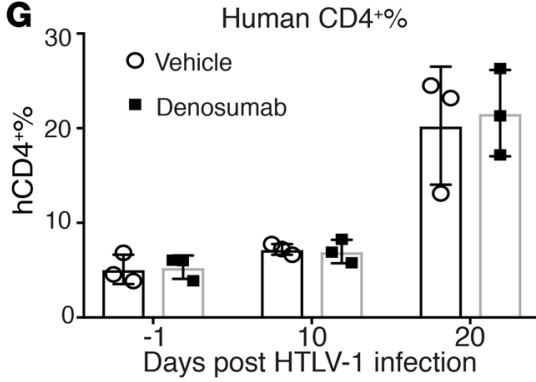

Figure 5. RANKL blockade with denosumab decreases disease-associated bone loss. (A) Experimental design: HTLV-1-infected humanized mice were treated with vehicle (PBS) or denosumab twice weekly for 3 weeks from day 3 before sacrifice. (B) $\mu$ CT analysis used to calculate trabecular bone to tissue volume ratio (BV/TV) on day 21. (C) Representative 3-D reconstruction images of tibial trabecular bone in a denosumab treated mouse compared with vehicle control. Scale bar: $300 \mu \mathrm{m}$. (D) $\mu \mathrm{CT}$ analysis used to calculate trabecular bone mineral density (BMD) on day 21. (E) Spleen weight measured on day 21. (F) Flow cytometry of human CD45 and (G) human CD4+ T cells from PBMC obtained before infection (day -1 ) and on days 10 and 20 after infection. Error bars in this figure represent $\pm \mathrm{SEM}$. ${ }^{*} P<0.05 ;{ }^{* *} P<0.01$ (2-tailed distribution, homoscedastic Student's $t$ test).

this dataset, HBZ-FLAG was transduced in mouse primary CD4+ $\mathrm{T}$ cells, and ChIP-seq was subsequently performed using a FLAG antibody. We found no peaks associated with the RANKL promoter and enhancer regions (Supplemental Figure $4 \mathrm{~A}$ ), suggesting that there was no direct interaction between $\mathrm{HBZ}$ and the $R A N K L$ promoter or enhancer regions. We then explored the hypothesis that $R A N K L$ gene expression is regulated by HBZ indirectly, using a candidate approach based on known regulators of $R A N K L$ expression. It has been shown that $R A N K L$ gene expression is regulated by c-Fos through a cluster of distal regulatory enhancers designated as the T cell control region (TCCR) in both human and mouse $\mathrm{T}$ cells $(37,38)$. To test the hypothesis that HBZ affects $R A N K L$ expression through regulation of c-Fos, we examined the $c$-Fos (FOS) gene expression in the HBZ expressing Jurkat cells by qPCR and found significant upregulation of c-Fos expression in the HBZ-expressing Jurkat cells, compared with the WT Jurkat cells (Figure 4D). In addition, we evaluated the expression of c-Fos in the Kit-225 human T cell line expressing WT HBZ and HBZ mutants (HBZ SM1 and $\triangle \mathrm{HBZ}$ ). We found that $c$-Fos gene expression was specifically upregulated by HBZ protein (SM1) in this system, and no induction of $c$-Fos gene expression was observed in the HBZ RNA-expressing mutant ( $\triangle \mathrm{HBZ}$ ). (Figure $4 \mathrm{E}$ ). HBZ and c-Fos are both bZIP transcription factors. It has been reported that HBZ interacts with c-Fos binding partner c-Jun; however, no direct HBZ/c-Fos protein-to-protein interaction was observed (39). Thus, we investigated whether HBZ protein could directly interact with the $c$-Fos promoter. We examined the HBZ-FLAG ChIP-seq and found no evidence for direct interaction between HBZ and c-Fos gene (Supplemental Figure 4A). In addition, using a c-Fos promoter reporter luciferase assay, we found no change in c-Fos promoter activity after the induction of HBZ in 
Jurkat cells (Supplemental Figure 4, B and C). Together, these data suggest that the upregulation of $c$-Fos in HBZ-expressing cells is likely indirectly regulated through other mediators.

Finally, we used the CRISPR/Cas9 system to delete the c-Fos (FOS) gene in HBZ-overexpressing Jurkat cells (Jurkat-HBZ). Briefly, Jurkat-HBZ cells were transduced with LentiCRISPRv2 vector either empty (CRISPRv2-Empty vector) or targeting the c-Fos genes (CRISPRv2-FOS KO) and selected with puromycin. Single cell clones were obtained by limiting dilution assay, and c-Fos protein levels in the selected clones were verified by Western blot (Supplemental Figure 4D). We found diminished levels of c-Fos protein in clone \#1 and complete protein $\mathrm{KO}$ in clones \#2 and \#3, even following exposure to phorbol12-myristate 13-acetate (PMA), a well-known inducer of c-Fos expression. By qPCR, we found a significant reduction in RANKL gene expression in the 3 CRISPR-FOS-KO clones of the Jurkat-HBZ cells (Figure 4, F and G). Taken together, these data suggest that HBZ induces the transcription of $R A N K L$ in a c-Fos-dependent manner.

RANKL blockade with denosumab decreases disease-associated bone loss. We next asked whether tumor cellderived RANKL is directly responsible for disease-associated bone loss. To answer this question in the HTLV1 infection model, we blocked RANKL with denosumab, a monoclonal antibody that specifically targets human RANKL while sparing host-derived murine RANKL (40). Denosumab is clinically used to treat bone metastases and osteoporosis (41). We confirmed that denosumab potently blocked human osteoclast differentiation and had little effect on murine osteoclast differentiation (Supplemental Figure 5A). Likewise, in vivo administration of denosumab had no effect on bone mass in non-tumor bearing, NSG mice (Supplemental Figure 5, B and C). We administered denosumab to HTLV-1-infected humanized mice twice weekly for 3 weeks at a dose of $25 \mathrm{mg} / \mathrm{kg}$, which is equivalent to the human treatment dose for bone metastases (Figure 5A). Treatment with denosumab reduced HTLV-1-infected mice from disease-associated bone loss (BV/TV) by $21 \%$ compared with the PBS-treated group, as measured by $\mu \mathrm{CT}$ (Figure $5, \mathrm{~B}$ and $\mathrm{C}$ ). BMD trended up in denosumab-treated humanized mice, although it did not reach statistical significance (Figure 5D). We did not observe significant effects of denosumab on disease burden (hCD4 ${ }^{+} \mathrm{T}$ cells) as measured by flow cytometry and spleen weight (Figure 5, E-G). Because denosumab only targets human RANKL, these data suggest that LPD-derived RANKL directly contributes to ATL-associated bone loss.

ATL patient-derived xenografts exhibit marked osteolytic bone loss and increased RANKL expression. To test if primary ATL cells from patients induced comparable osteolytic bone loss in vivo, we inoculated freshly obtained, $\mathrm{CD}^{+}$sorted tumor cells from ATL patients into NSG mice with mutant Kit (NBSGW mice) by i.p. injection. NBSGW mice have significant hematopoietic defects due to the c-Kit mutation and do not require irradiation prior to human cell engraftment, in order to prevent rejection. The primary ATL cells had never been frozen or expanded in cell culture prior to inoculation (Figure 6A). ATL patient derived xenografts (PDX) were derived from 2 independent patients with acute ATL (ATL-PDX-01 and ATL-PDX-02), one (ATL-PDX-02) of whom was clinically diagnosed with hypercalcemia. Gene expression analysis demonstrated that ATL cells from both patients expressed Tax and HBZ transcripts (Supplemental Figure 6, A and B). Probe-capture-based, targeted next-generation DNA sequencing revealed that ATL cells from the 2 patients carried different driver mutations and had distinct clonality profiles, confirming their genetic diversity (Supplemental Figure 6, C and D). Necropsy was performed 9 weeks after tumor cell injection and revealed enlargement of the spleen, lung, and liver in mice injected with both ATL-PDX clones compared with the control NBSGW mouse (Figure 6, B and C). Flow cytometry performed on cells from organs (spleen and liver) and peripheral blood revealed the apparent organ infiltration of $\mathrm{CD} 45^{+} \mathrm{CD} 4^{+}$tumor cells in the spleen and liver tissues ( $\geq 30 \%$ ), while peripheral blood was less involved ( $>5 \%$ ) (Figure $6 \mathrm{D}$ ). Notably, blood smear from mice inoculated with ATL-PDX-02 showed the "flower cells" characteristic of ATL (Figure 6E). Importantly, mice injected with either of the ATL-PDX clones exhibited significant osteolytic bone loss compared with an age-matched controls, with markedly decreased BV/TV and BMD by $\mu \mathrm{CT}$ (Figure 6, F-H). We next evaluated the expression of FOS and RANKL in the ATL-PDX-01, and we found that both FOS and RANKL gene expression was significantly elevated in ATL-PDX cells compared with T cells isolated from PBMC of healthy donors (Figure 6, I and J). It has been reported that alternate RANKL transcripts encode distinct membrane and secreted RANKL isoforms (42). We detected transcripts of the membrane-bound, but not the secreted, RANKL isoform in human PBMC, as well as the ATL-PDX-01 cells (Supplemental Figure 7). These data demonstrate that the ATL-PDX mice model the clinical features of ATL including "flower cells," hepatosplenomegaly, and bone loss. Increased FOS and RANKL gene expression further suggests that this bone-modifying pathway is hyperactivated in human ATL. 
A

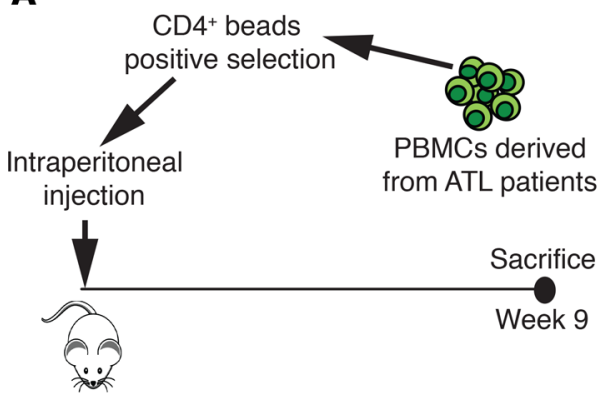

B

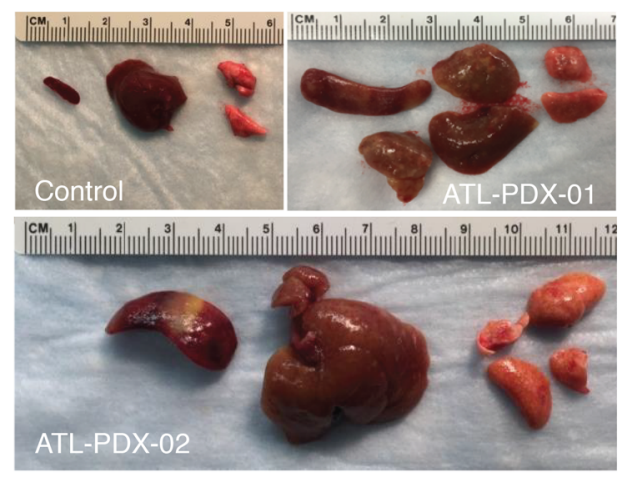

C

Spleen weight

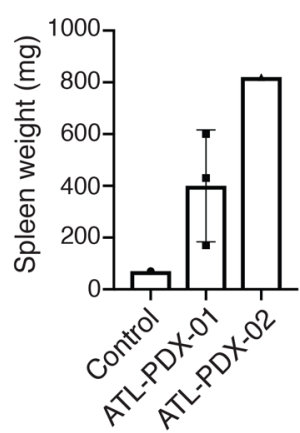

\section{D}
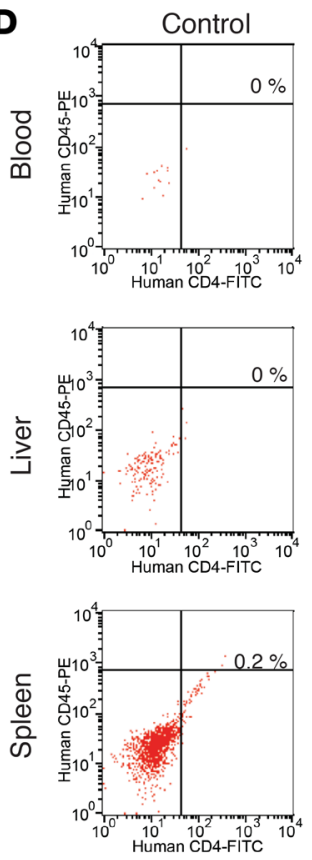

H

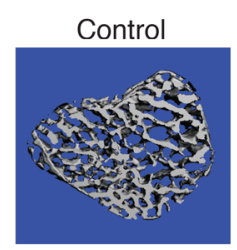

ATL-PDX-01
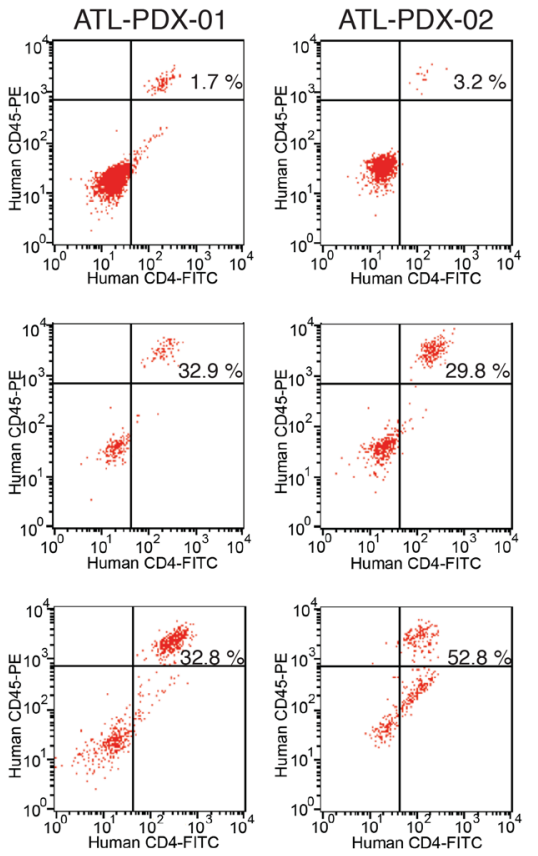

$\mathbf{F}$

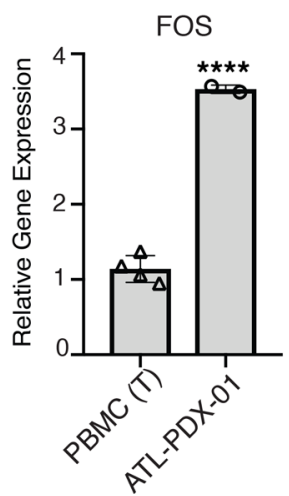

E

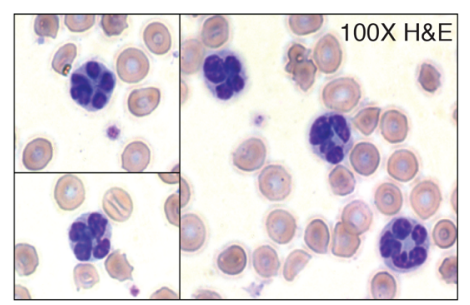

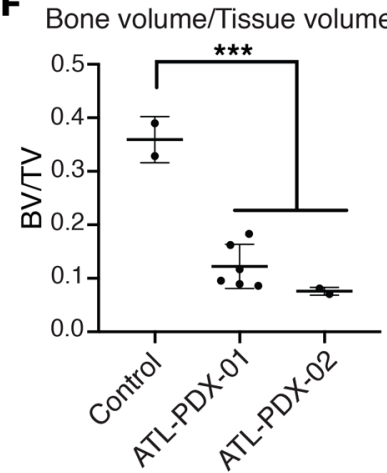

I

F Bone volume/Tissue volume

G Bone mineral density

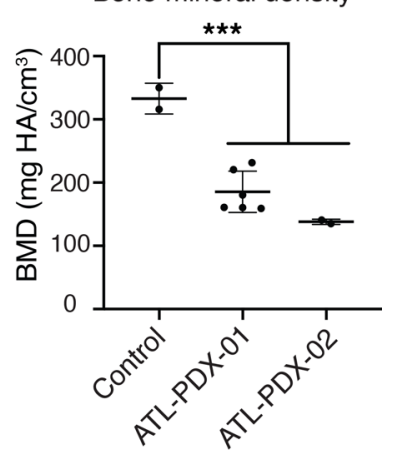

J

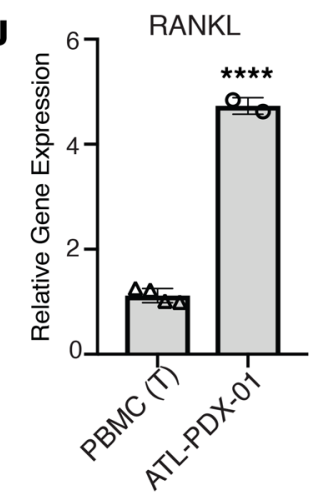

Figure 6. ATL patient-derived xenografts exhibit marked osteolytic bone loss and increased RANKL expression. (A) Experimental design: CD4 ${ }^{+}$ primary tumor cells derived from the peripheral blood of 2 acute ATL patients (ATL-PDX-01 and ATL-PDX-02) were purified and injected into NBSCW mice by i.p. injection; 1 uninjected NBSGW mouse was used as control; mice were sacrificed at week 9 after inoculation ( $n=1-3$ per group). (B) ATL-PDX-bearing mice presented with enlargement of spleen, liver, and lung. (C) Increased spleen mass is shown. (D) Flow cytometry for human CD45+CD4+ ATL cells in the blood and spleen. (E) The presence of "flower cells" in peripheral blood smears in mice inoculated with ATL-PDX-02 cells. (F and $\mathbf{G}) \mu C T$ analysis for calculation of tibial trabecular bone to tissue volume ratio (BV/TV) and bone mineral density (BMD). (H) Representative images of 3-D reconstruction of tibial trabecular bone from control and ATL-PDX mice. Scale bar: $500 \mu \mathrm{m}$. (I) In vitro qPCR detection of FOS and (J) RANKL RNA in primary ATL cells (ATL-PDX-01), compared with T cells from PBMCs. Data is representative of 2 biological replicates. Error bars in this figure represent $\pm \mathrm{SEM} .{ }^{*} P<0.05 ;{ }^{* *} P<0.01$; ${ }^{* * *} P<0.001 ;{ }^{* * *} P<0.0001$ (2-tailed distribution, homoscedastic Student's $t$ test for 2 groups or 1 -way ANOVA for multiple comparison). 


\section{Discussion}

Osteolytic bone lesions and hypercalcemia are common and significant complications in patients with ATL (4). Both of the HTLV-1 viral oncogenes Tax and HBZ have been shown to independently regulate expression of cellular genes involved in osteoclast activation and development. However, in the context of viral infection and subsequent oncogenic transformation, the role of each viral oncogene in clinical bone loss has not been defined. It has been shown that the HTLV-1 Tax oncogene regulates several bone-activating factors, and we previously found that Tax drives bone loss and hypercalcemia in Tax transgenic mice (8). Despite these findings, Tax gene expression is very low in the majority of the ATL patients with advanced leukemia and skeletal complications, prompting consideration of other drivers of bone destruction in ATL patients. In contrast to Tax loss over time, HBZ is consistently expressed throughout ATL development and progression (11). HBZ expression has also been shown to modulate osteoblast regulators such as DKK1 and Wnt5a $(43,44)$, and we recently reported that aged Gzmb-HBZ transgenic mice develop LPD associated with bone loss and hypercalcemia (19). These data suggest that Tax and HBZ can independently regulate bone-acting factors and could contribute to disease-associated bone loss.

In the current study, we sought to investigate the role of HBZ in ATL-associated bone loss in a HTLV-1 infection model in CD34+ humanized mice. We found that HTLV-1-infected mice had continuous bone loss and increased osteoclast activity over the course of disease development, proportional to hCD4 ${ }^{+} \mathrm{T}$ cell burden in the peripheral blood. Infection with HTLV-1 $\triangle \mathrm{HBZ}$, a mutant virus with a stop codon point mutation, still induced LPD (albeit less than WT virus) but exhibited no bone loss despite the development of LPD, suggesting that HBZ protein may play an important role in ATL bone disease. We found that RANKL, a critical factor for osteoclastogenesis and bone homeostasis, was upregulated by HBZ. Selective blockade of human RANKL by the clinical RANKL antagonist, denosumab, decreased disease-associated bone loss in HTLV-1-infected humanized mice.

In our current humanized mouse model, we found that mice infected with HTLV-1 $\triangle \mathrm{HBZ}$ developed progressive splenomegaly and fatal LPD, though with delays in disease onset. Because HBZ RNA is not altered in the $\triangle \mathrm{HBZ}$ mutant, we cannot rule out the possibility that HBZ RNA contributed to the transformation and proliferation process in the absence of $\mathrm{HBZ}$ protein, and it could have partially rescued or modulated disease development that was observed in this mutant lacking HBZ protein, although all mice infected with HTLV-1 $\triangle \mathrm{HBZ}$ died of LPD (Figure 1). In order to account for differences in disease burden and resultant effects on bone loss, we quantitated the number of circulating hCD $4^{+}$cell counts compared with bone mineral content and osteoclast number for each group of mice. $\mathrm{CD} 4^{+}$cell counts predictably increase in this HTLV-1-infected humanized mice model as disease and spleen size increases. We found that increasing $\mathrm{CD} 4^{+}$counts correlated with increased osteoclast number and decreased BMD in HTLV-1-infected mice, but no such correlation was observed in HTLV-1 $\triangle \mathrm{HBZ}$ infected mice, despite mice developing LPDs. The complete uncoupling of disease burden and osteolysis with $\triangle \mathrm{HBZ}$ infection suggests that $\mathrm{HBZ}$ itself plays a role in bone loss.

T cell-derived RANKL has been shown to mediate bone loss in arthritis and periodontal disease (24, 45). Similar to previous reports that RANKL is elevated in ATL patients with hypercalcemia (25), we detected elevated RANKL levels in ATL-PDX cells derived from an ATL patient. ATL-PDX cells, like human PBMC, express only the transcript for membrane-bound RANKL, but not soluble RANKL, consistent with the reported requirement for cell-to-cell contact in cocultures of ATL cells to induce osteoclasts (25). ATL cells can reside in BM, which could facilitate the delivery of membrane-bound RANKL to adjacent resident osteoclasts and their precursors. In our previously published work, we found that Tax overexpression increased osteoclast-activating factors such as IL-6 and TNF- $\alpha$, but not RANKL (8). Likewise, we did not observe increased RANKL after the induction of Tax in T cells using a Tet-on Tax1 system (Supplemental Figure 3, A and B). Based on these findings, we investigated whether HBZ could regulate RANKL. We used constructs enabling selective expression of either HBZ protein (SM1) or RNA ( $\triangle \mathrm{HBZ}$ ) in T cells to dissect their respective contributions to control of $R A N K L$ expression. HBZ protein and RNA have been shown to have distinct functions, with HBZ RNA itself playing an independent role in cell proliferation and survival $(17,18)$. We found that HBZ protein, but not HBZ RNA, drives the expression of RANKL. HBZ ChIP-seq data further revealed that HBZ protein does not directly interact with the RANKL gene promoter or distant enhancers, suggesting that the upregulation of $R A N K L$ gene expression was indirect. Because c-Fos is a well-established regulator of RANKL expression in T cells $(37,38)$, we next looked at c-Fos levels and found that HBZ protein upregulated c-Fos gene expression in T cells and that CRISPR deletion of c-Fos blocked HBZ upregulation of RANKL. Further examination of the HBZ-FLAG ChIP-seq data and 
promoter reporter assay suggests that the upregulation of c-Fos by HBZ is likely indirectly regulated through other mediators. In line with the data that HBZ regulates the osteoclast-acting factors RANKL and c-Fos, we found by histomorphometry that humanized mice infected with HTLV-1 $\triangle$ HBZ had significantly lower osteoclast numbers compared with HTLV-1-infected mice. Further studies are needed to explore the mechanisms through which c-Fos is upregulated by HBZ.

There are numerous preclinical models of ATL, each having strengths and weaknesses with regard to their successful recapitulation of human ATL and ability to specifically evaluate HBZ in HTLV-1 transformation and disease progression. We reported that transgenic mice overexpressing HBZ in activated T cells developed LPD and osteolytic bone disease at 18 months (19); however, HBZ expression in this model is genetically driven outside the context of HTLV-1 infection. We have previously evaluated the role of HBZ in viral infection using an immunocompetent rabbit HTLV-1 infection model, which has been extensively used to study HTLV-1 viral transmission and infection (28). We found that loss of HBZ in this system resulted in a decreased host immune response to viral infection and a decreased proviral load compared with the WT-infected animals (16). However, the rabbit HTLV-1 infection model does not induce LPD. In order to examine the role of HBZ in a model that can sufficiently replicate the clinical features of ATL using HTLV-1 WT or HBZ mutant viruses, we used the CD34 ${ }^{+}$humanized mouse model, which enables rapid reconstitution of human lymphoid cells and subsequent development of leukemia-like disease following HTLV-1 infection (32). This is a robust system to study HTLV-1-induced LPD and bone disease in the context of the HTLV-1 but still has few limitations with regard to the role of immune responses during ATL transformation and progression. The reconstitution of myeloid cells is less efficient compared with lymphoid cells in this model (46). In addition, the T cells in this model are educated in the murine thymus, thus restricted to H2-major histocompatibility complex (MHC) (29). This lack of both innate and adaptive host immune responses to ATL in HTLV-1-infected humanized mice is less than optimal. Future studies are underway to use transgenic humanized mice expressing human IL-3 and IL-13 that can support the development of human cells derived from the myeloid lineage such as macrophage cells and DCs (47). Expression of human MCSF in mice by hydrodynamic delivery of cytokine-encoding plasmids through tail vein injection would also promote the induction and development of functional human macrophages in a humanized mouse model (48). We will also explore the use of a BM/liver/thymus (BLT) humanized mouse model, in which the $\mathrm{T}$ cells are educated in an autologous transplanted human thymus and are human leukocyte antigen (HLA) restricted $(49,50)$

Similar to HIV-1, HTLV-1 infection is lymphotropic and predominantly transforms CD4 ${ }^{+}$T cells. However, both HIV-1 and HTLV-1 virus can infect multiple cell types other than T cells, including DCs, macrophages, and mesenchymal stromal cells $(51,52)$. Recently, it has been demonstrated that HIV-1 virus can infect osteoclasts directly and could induce enhanced adhesion and osteolytic activity of the osteoclasts through the HIV-1 viral protein Nef (53). While HTLV-1 can infect macrophages (51), it is unknown whether HTLV-1 can infect osteoclasts and affect their osteolytic functions. We are currently working on selectively expressing HBZ in the myeloid lineage (osteoclast) cells to evaluate effects on bone loss and resorption using in vivo models.

Denosumab, a monoclonal antibody that targets human RANKL, is the standard of care for patients with osteoporosis, hypercalcemia of malignancy, and bone metastases (54). Denosumab selectively targets human RANKL and does not bind to mouse RANKL (40). In our study, we found that denosumab, which only targeted human cell-derived RANKL, resulted in decreased HTLV-1 LPD-induced bone loss. In this study, we used the dose equivalent to treatment dose in human patients ( $120 \mathrm{mg} /$ dose). We have also tested osteoclastogenesis in vitro and confirmed that denosumab had no effect on mouse osteoclast formation and no effect on mouse BMD in vivo, but it inhibited human osteoclast formation at low nanomolar concentration. Because denosumab did not completely protect mice from HTLV-1 disease-induced bone loss, this suggests that other tumor-associated factors can contribute. Currently, zoledronic acid is more commonly used than denosumab as the first line to prevent bone loss and fractures in patients with multiple myeloma, the other hematologic malignancy with high rates of bone destruction (55). Our findings that denosumab decreased disease-associated bone loss in HTLV-1-infected humanized mice support the conclusion that human-derived RANKL drives bone loss in this model and suggests that denosumab should be considered for ATL patients to prevent or decrease tumor-associated bone destruction.

Currently, there are no models of ATL that recapitulate all aspects of HTLV-1 ATL pathogenesis. The HTLV-1-infected humanized model used in this paper does not develop overt hypercalcemia, so we cannot extrapolate the effects of HBZ on bone loss to hypercalcemia. There have been reports using primary ATL 
cells in immunodeficient mice $(56,57)$; however, none has examined the ATL-associated bone disease in patient-derived xenograft models. We used $\mathrm{CD}^{+}$cells purified from 2 patients with acute ATL and inoculated in NBSGW immunodeficient mice. We observed engraftment and rapid expansion of malignant cells, with replication of important clinical features of acute ATL in vivo, including organ and blood involvement, with circulating blasts with the "flower cell" morphology. We report for the first time to our knowledge that the ATL-PDX mice also develop significant osteolytic bone destruction, as is commonly observed in patients. ATL-PDX-02 was derived from a patient with hypercalcemia, one of the classic skeletal complications frequently observed in patients with ATL. While no clinical signs of hypercalcemia was observed in the patient, ATL-PDX-01 also induced bone loss in vivo. Although the degree of bone loss in the 2 PDX lines was similar at the endpoint, it is possible that different mechanisms are present in hypercalcemic vs. nonhypercalcemic patients, and these could be identified by comparing multiple PDX from the 2 patient types. ATL-PDX models could be used to gain insights into the biological impact on bone disease in naturally occurring HTLV-1 mutants.

In conclusion, we demonstrate that, in HTLV-1-infected humanized mice that develop LPD, bone destruction occurs early during malignant transformation and progressively worsens with disease burden. This bone loss was associated with increased osteoclast activity and correlated with LPD burden. We found that the viral oncoprotein HBZ, through upregulation of RANKL, is a key factor responsible for ATL-associated bone loss and that RANKL inhibition with denosumab could be useful to treat and to prevent pathologic bone loss in patients with ATL. Using primary CD4 ${ }^{+}$human ATL cells derived from 2 ATL patients to induce LPD, we also observed profound tumor-induced bone destruction and increased $c$-Fos and $R A N K L$ gene expression. Our study shows that HTLV-1-infected humanized mice and primary human ATL-PDX models can be used to study molecular mechanisms underlying bone disease in ATL, as well as to develop novel therapies for the treatment of ATL-associated bone disease.

\section{Methods}

Cell lines and reagents/drugs. Human T cell leukemia lines Jurkat and Kit-225 (ATCC) were maintained in RPMI 1640 supplemented with 10\% FBS. Kit-225 cells were cultured in the presence of $20 \mathrm{U} / \mathrm{ml}$ of human recombinant IL-2 (Peprotech). Tet-on Tax1 Jurkat cells were maintained in RPMI 1640 supplemented with $10 \%$ FBS; $1 \mu \mathrm{g} / \mathrm{ml}$ doxycycline was used to induce Tax expression, as previously described (35). A human B-lymphoblastoid cell line, 729 cells, were transfected with a molecular clone of HTLV-1 (729.HTLV-1) or a molecular clone of HTLV-1 with deletion of HBZ protein (729.HTLV-1 $\triangle H B Z$ ) (16). HTLV-1-producing cells 729.HTLV-1 and 729.HTLV-1 $\triangle$ HBZ were maintained in IMDM supplemented with $10 \%$ FBS as previously described (58). Experiments involving HTLV-1 were performed under biosafety level 2 practices and procedures.

Animal studies. NSG mice and C57BL/B6 mice were obtained from The Jackson Laboratory. Humanized mice were generated by intrahepatic injection of sublethally irradiated (100 cGy) NSG neonatal mice with $3 \times 10^{4}$ to $1 \times 10^{5} \mathrm{hCD} 34^{+}$umbilical cord blood cells (Lonza) as described previously (31). At week 10, the efficiency of humanization was evaluated by the presence of $>15 \% \mathrm{hCD} 45^{+}$cells in peripheral blood. Mice were subsequently infected with HTLV-1 by i.p. inoculation of $1 \times 10^{7}$ lethally irradiated (100 cGy) HTLV-1producing cells (729.HTLV-1 WT or 729.HTLV-1 $\triangle$ HBZ). Blood, long bones, and spleens were obtained from HTLV-1-infected humanized mice, which were analyzed at 1, 3, and 5 weeks after infection. To control for sex differences in bone physiology, male mice were used for the bone parameter analyses.

Denosumab (trade name Prolia) was obtained from Amgen. In the denosumab studies, HTLV-1-infected humanized mice or uninfected NSG mice were treated from day 3 after infection with vehicle (PBS) or denosumab (25 mg/kg, s.c. injection) twice weekly for 3 weeks before sacrifice.

Primary ATL patient-derived xenograft model. Primary ATL tumor cells were isolated using CD4 magnetic microbeads (Miltenyi Biotec) from PBMCs freshly obtained from 2 acute ATL patients with informed consent.Purified CD4 ${ }^{+}$ATL cells $\left(5 \times 10^{6}\right.$ cells) were inoculated in 7-week-old NBSGW (NOD.Cg-Kit ${ }^{\mathrm{W}-41 \mathrm{~J}}$ $\mathrm{Tyr}^{+}$Prkdc $^{\text {scid }} \mathrm{Il2} \mathrm{rg}^{\mathrm{tm} 1 \mathrm{Wj}} / \mathrm{ThomJ}$ ) mice (The Jackson Laboratory) by i.p. injection. Mice housed under pathogen-free conditions were monitored for weight loss, dehydration, or other signs of morbidity. Blood, spleen, lung, and liver tissue were harvested from humanely euthanized mice, disaggregated, filtered, and stained for flow cytometry analysis to quantify the hCD $45^{+} \mathrm{hCD} 4^{+}$ATL cells. Primary ATL tumor cells from the 2 acute ATL patients were also cultured in RPMI medium supplemented with 10\% FBS and cultured in the presence of $50 \mathrm{U} / \mathrm{ml}$ of human recombinant IL-2 (Peprotech). 
$\mu C T$. Tibiae were removed postmortem, scanned in a 17-mm holder using $\mu \mathrm{CT}$ ( $\mu \mathrm{CT}-40$; Scanco Medical), and evaluated by a blinded operator as described previously. A 3-dimensional cubical voxel model of bone was built, and calculations were made for BV/TV and apparent BMD (calibrated against a hydroxyapatite phantom) (59).

Bone histomorphometry and analysis. Mouse tibiae from the animals were fixed in $10 \%$ formalin for 24 hours and decalcified in 14\% EDTA solution for 2 weeks. Paraffin-embedded sections were stained with TRAP for osteoclast parameter analysis. Sections were analyzed by a blinded operator according to a standard protocol using Bioquant Osteo 2010 (Bioquant Image Analysis Corp.).

Plasmid and vectors. HBZ cDNA was cloned into the $\mathrm{pCDH}-\mathrm{CMV}-\mathrm{MCS}-\mathrm{T} 2 \mathrm{~A}-\mathrm{copGFP}$ lentiviral expression vector (SBI) and used to generate the Jurkat-HBZ cell line. HBZ cDNA was cloned into the pCI-neo mammalian expression vector (Promega) and used to generate the Kit-225-HBZ cell line. pCI-neo- $\Delta \mathrm{HBZ}$ was generated from pCI-neo-HBZ by introducing a $\mathrm{G}$ to $\mathrm{A}$ point mutation that resulted in termination of the HBZ reading frame at amino acid 8 , and it subsequently used to generate the Kit-225- $\Delta \mathrm{HBZ}$ cells. Previous studies have shown that the introduction of the $\triangle \mathrm{HBZ}$ mutation did not alter $\mathrm{p} 13$ and $\mathrm{p} 30$ viral protein expression (16). pCI-neo-HBZ SM1 was generated from pCI-neo-HBZ construct by introducing a $\mathrm{G}$ to A point mutation that resulted in the termination of the HBZ reading frame at amino acid 8 and subsequently used to generate the Kit-225-HBZ SM1 cells. Previous studies have shown that the introduction of the DHBZ mutation did not alter p13 and p30 viral protein expression (16). pCI-neo-HBZ SM1 was generated from pCI-neo-HBZ construct by introducing a silent mutation in the third nucleotide position of the first 210 amino acids. This construct alters the RNA secondary structure and was used to generate Kit-225-HBZ SM1 cells.

CRISPR/Cas9 KO of FOS gene. LentiCRISPRv2 was a gift from Feng Zhang (Addgene plasmid 52961). Individual targeting gRNA for FOS gene (5'-CTGCAGCCAAATGCCGCAAC-3') was cloned into the LentiCRISPRv2 plasmid as previously described (60). Lentivirus production and infection was performed as described previously (61). Transduced Jurkat-HBZ cells were selected with $1 \mu \mathrm{g} / \mathrm{ml}$ puromycin (Sigma-Aldrich) for 3-5 days. Single clones of transduced HBZ-expressing Jurkat cells were obtained by standard limiting dilution assay. Western blot was performed to confirm the efficiency of FOS KO.

Western blot. Cells were harvested and lysed with Laemmli Sample Buffer (Bio-Rad). Whole cell lysates of the cells were then separated on $8-12 \%$ SDS-polyacrylamide gels (Bio-Rad), transferred onto polyvinylidene difluoride membranes, and incubated with c-Fos (Cell Signaling Technology, 4384) or HSP90 antibodies (Enzo Life Sciences, ADI-SPA-836-D), followed by horseradish peroxidase-conjugated anti-rabbit secondary antibody (Cell Signaling Technology, 7074). All antibodies were diluted and used according to the manufacturers' protocol.

$q P C R$ and primers. RNA was extracted from cells using RNeasy kit (Qiagen), and cDNA was generated with qScript cDNA SuperMix (Quanta Bio). qPCR was completed using PerfeCTa SYBR Green SuperMix Reagent (Quanta Bio) on the BioRad CFX96 machine (Bio-Rad). All procedures were performed according to the instruction from the manufacturer. Primers designed for qPCR are: RANKL (forward: 5' - CCCAAGTTCTCATACCCTGATG - 3', reverse: 5' - TTCCTCTCCAGACCGTAACT - 3'; FOS (forward: 5' - GGACTCAAGTCCTTACCTCTTC - 3', reverse: 5' - CCTGGCTCAACATGCTACTA - 3'; GAPDH (forward: 5' - TGTGATGGGTGTGAACCACGAGAA - 3', reverse: 5' - GAGCCCTTCCACAATGCCAAAGTT - 3').

Proviral load assessment by digital droplet PCR (ddPCR). Genomic DNA was isolated from the spleen tissue of HTLV-1-infected humanized mice. HTLV-1 Tax (154 bp) and a human housekeeping gene, RPP30 (62 bp), were amplified as follows: The ddPCR assays were performed by mixing $0.9 \mu \mathrm{M}$ primers, 0.25 $\mu \mathrm{M}$ probe, $2 \times$ ddPCR Supermix for Probes (Bio-Rad) and $100 \mathrm{ng}$ extracted DNA in a final volume of $20 \mu 1$. Droplets were generated in the QX-200 droplet generator (Bio-Rad) according to manufacturer's instructions. Droplets were transferred to a 96-well PCR plate, and the subsequent PCR amplification was performed in the thermal cycler CFX96 (Bio-Rad) with these cycling parameters: $95^{\circ} \mathrm{C}$ for 10 minutes, 40 cycles of $94^{\circ} \mathrm{C}$ for 30 seconds, $60^{\circ} \mathrm{C}$ for 1 minute, 10 minutes at $98^{\circ} \mathrm{C}$, and a hold at $4^{\circ} \mathrm{C}$. After PCR amplification, the droplets were analyzed in the QX200 droplet reader (Bio-Rad). The results were visualized with the software QuantaSoft version 1.6.6 (Bio-Rad). Each sample was analyzed in 2 wells, and for each sample, a no template control (NTC) was added. Based on the background in the NTC, a threshold was set manually just above the background in order to define positive droplets.

Based on this threshold, the software calculated the absolute concentration for each sample. The PVL was calculated using the following formula: 
$\left(\frac{\text { Quantity of HTLV-1 Tax }}{\left(\frac{\text { Quantity of housekeeping gene }}{2}\right)}\right) \times 100$

Human and mouse osteoclast differentiation assay. For mouse osteoclast differentiation, BM macrophages were harvested and cultured in $\alpha$ MEM with $10 \%$ FBS and supplemented with $100 \mathrm{ng} / \mathrm{ml}$ MCSF for 3 days. Then, the macrophages were cultured in osteoclast differentiation media ( $\alpha \mathrm{MEM}, 10 \% \mathrm{FBS}, 50 \mathrm{ng} / \mathrm{ml}$ $\mathrm{MCSF}$, and $50 \mathrm{ng} / \mathrm{ml}$ mouse RANKL) for 5 days.

For human osteoclast differentiation, $\mathrm{CD}_{14}{ }^{+}$monocytes were first purified from human PBMCs of healthy donors using $\mathrm{CD} 14^{+}$beads (Miltenyi Biotec). Purified CD $14^{+}$monocytes were cultured in osteoclast differentiation media ( $\alpha$ MEM, 10\% FBS, $40 \mathrm{ng} / \mathrm{ml} \mathrm{MCSF}$, and $40 \mathrm{ng} / \mathrm{ml}$ human RANKL) for 5 days. In both mouse and human osteoclast differentiation assays, various concentration of denosumab or PBS were added at the same time as the mouse/human RANKL. Media was refreshed every 2 days. Cells were fixed and stained for TRAP using the leukocyte acid phosphatase kit (MilliporeSigma).

Flow cytometry. Whole blood was collected from the submandibular vein into a capillary blood microtainer containing EDTA (BD Biosciences, catalog 365974). RBCs were lysed by using Gibco AKC Lysis buffer (Thermo Fisher Scientific, catalog A1049201). Samples were stained with commercially available fluorophore-labeled monoclonal antibodies specific for various leukocyte populations: CD45 (clone HI30, catalog MHCD4530TR), hCD3 (clone S4.1, catalog MHCD0327), CD4 (clone S3.5, catalog MHCD0420TR), and CD8 (clone 3B5, catalog MHCD0804) (Invitrogen). Four-color immunophenotyping of whole blood was performed on an Attune NxT Flow cytometer (Invitrogen). For primary ATL patientderived xenografts, PE mouse anti-hCD45 (clone HI30, catalog 555483) and FITC mouse anti-hCD4 (clone RPA-T4, catalog 555346) (BD Pharmingen) antibodies were used.

Microarray and ChIP-seq data analysis. For the ATL patient microarray expression data, we used publicly available data at NCBI GEO database with accession number GSE33615 $(34,63)$. We analyzed the microarray data according to the instructions from NCBI GEO2R (64). In the analysis, we compared differentially expressed genes between $\mathrm{CD}^{+} \mathrm{T}$ cells from 21 normal healthy donors and PBMC from 26 acute ATL patients (mostly HTLV-1-infected CD4 ${ }^{+}$T cells).

Publicly available HBZ ChIP-seq data was obtained from DNA Data Bank of Japan (DDBJ) under accession number DRA003229 (36). ChIP-seq data was mapped to the murine reference genome and analyzed as previously described using Bowtie and MACS $(65,66)$. Peaks were visualized using UCSC genome browser (67).

Statistics. Experiments were analyzed using 2-tailed Student $t$ test (2 groups), 1-way ANOVA ( $>2$ groups or repeated measures), or 2-way ANOVA (2 variables, $P$ value refers to the interaction) using Prism 8 (GraphPad Software Inc.). Pearson correlation coefficient test was adopted to determine statistically significant correlation between the 2 groups. Results were considered to reach significance at $\mathrm{P} \leq 0.05$ and are indicated with asterisks $\left({ }^{*} P<0.05 ;{ }^{*} P<0.01 ; * * * P<0.001 ; * * * * P<0.0001\right)$. Data are presented as mean values; error bars represent \pm SEM. Mouse weight changes are presented as mean and range. Survival (Kaplan-Meier) curves and median survival were analyzed in Prism 8.

Study approval. Experiments involving HTLV-1 were performed under BSL2 practices and procedures. Animals were housed under pathogen-free conditions according to the guidelines of the IACUC of Washington University and Ohio State University. All animal experiments were approved by the IACUC of Washington University and Ohio State University.

\section{Author contributions}

JX, DAR, DDH, SN, LR, and KNW conceived and designed the study. JX, DAR, DDH, ARP, XC, JCH, PLG, SN, LR, and KNW developed the study methodology. JX, DAR, DDH, ARP, XC, JCH, XS, YX, JS, WHW, YJ, SN, LR, and KNW acquired data. JX, DAR, DDH, SN, LR, and KNW analyzed and interpreted data. JX, DAR, DDH, ARP, AKE, GCF, FF, TK, HS, TJR, DJV, PLG, SN, LR, KNW were involved in the writing, review, and revision of the manuscript.

\section{Acknowledgments}

The authors thank Vivek Arora, Steven Teitelbaum, Wei Zou, and Ricardo Ramirez for their valuable expert suggestions and criticism. The authors thank Crystal Idleburg, Ancy Joseph, Nicole Kohart, Wessel 
Dirksen, and Kevin Wu for their technical assistance. The authors also thank the Musculoskeletal Research Center for histology and microCT (NIH P30-AR057235), the Hope Center Alafi Neuroimaging Lab for use of the Nanozoomer (NIH shared Instrumentation grant S10RR027552), Barnes-Jewish Hospital Foundation, the Siteman Cancer Center, and St. Louis Men's Group Against Cancer.

This research was supported by Program Project Grants from NIH PO1 CA100730 (to PLG, KNW, and LR), CA154737 (KNW), CA097250 (KNW), CO1 CA063417 (LR, DAR, XC, JH, and HS), P30 CA91842 (LR, DAR, XC, JH, and HS), R01 AR070030 (DJV), the Shriners' Hospitals for Children(DJV), 5T32CA113275-07 (AKE), and GM07200 (GCF). Additional funding support was provided by grants from the St. Louis Men's Group Against Cancer, Barnes-Jewish Hospital Foundation, and the Siteman Cancer Center.

Address correspondence to: Katherine N. Weilbaecher, Campus Box 8069, 660 South Euclid Avenue, St. Louis, Missouri 63110, USA. Phone: 314454-8858; Email: kweilbae@wustl.edu.

1. Gessain A, Cassar O. Epidemiological Aspects and World Distribution of HTLV-1 Infection. Front Microbiol. 2012;3:388.

2. Manel N, Battini JL, Taylor N, Sitbon M. HTLV-1 tropism and envelope receptor. Oncogene. 2005;24(39):6016-6025.

3. Shimoyama M. Diagnostic criteria and classification of clinical subtypes of adult T-cell leukaemia-lymphoma. A report from the Lymphoma Study Group (1984-87). Br J Haematol. 1991;79(3):428-437.

4. Katsuya H, et al. Treatment and survival among 1594 patients with ATL. Blood. 2015;126(24):2570-2577.

5. Giam CZ, Semmes OJ. HTLV-1 Infection and Adult T-Cell Leukemia/Lymphoma-A Tale of Two Proteins: Tax and HBZ. Viruses. 2016;8(6):E161.

6. Grassmann R, Aboud M, Jeang KT. Molecular mechanisms of cellular transformation by HTLV-1 Tax. Oncogene. 2005;24(39):5976-5985.

7. Matsuoka M, Jeang KT. Human T-cell leukemia virus type 1 (HTLV-1) and leukemic transformation: viral infectivity, Tax, HBZ and therapy. Oncogene. 2011;30(12):1379-1389.

8. Gao L, et al. HTLV-1 Tax transgenic mice develop spontaneous osteolytic bone metastases prevented by osteoclast inhibition. Blood. 2005;106(13):4294-4302.

9. Hasegawa $\mathrm{H}$, et al. Thymus-derived leukemia-lymphoma in mice transgenic for the Tax gene of human T-lymphotropic virus type I. Nat Med. 2006;12(4):466-472.

10. Ohsugi T. A transgenic mouse model of human T cell leukemia virus type 1-associated diseases. Front Microbiol. 2013;4:49.

11. Matsuoka M, Jeang KT. Human T-cell leukaemia virus type 1 (HTLV-1) infectivity and cellular transformation. Nat Rev Cancer. 2007;7(4):270-280.

12. Furukawa Y, Kubota R, Tara M, Izumo S, Osame M. Existence of escape mutant in HTLV-I tax during the development of adult T-cell leukemia. Blood. 2001;97(4):987-993.

13. Takeda S, et al. Genetic and epigenetic inactivation of tax gene in adult T-cell leukemia cells. Int J Cancer. 2004;109(4):559-567.

14. Taniguchi Y, et al. Silencing of human T-cell leukemia virus type I gene transcription by epigenetic mechanisms. Retrovirology. 2005;2:64.

15. Matsuoka M, Green PL. The HBZ gene, a key player in HTLV-1 pathogenesis. Retrovirology. 2009;6:71.

16. Arnold J, et al. Enhancement of infectivity and persistence in vivo by HBZ, a natural antisense coded protein of HTLV-1. Blood. 2006;107(10):3976-3982.

17. Satou Y, Yasunaga J, Yoshida M, Matsuoka M. HTLV-I basic leucine zipper factor gene mRNA supports proliferation of adult T cell leukemia cells. Proc Natl Acad Sci USA. 2006;103(3):720-725.

18. Mitobe Y, Yasunaga J, Furuta R, Matsuoka M. HTLV-1 bZIP Factor RNA and Protein Impart Distinct Functions on T-cell Proliferation and Survival. Cancer Res. 2015;75(19):4143-4152.

19. Esser AK, et al. HTLV-1 viral oncogene HBZ induces osteolytic bone disease in transgenic mice. Oncotarget. 2017;8(41):6925069263.

20. Novack DV, Teitelbaum SL. The osteoclast: friend or foe? Annu Rev Pathol. 2008;3:457-484.

21. Long F. Building strong bones: molecular regulation of the osteoblast lineage. Nat Rev Mol Cell Biol. 2011;13(1):27-38.

22. Weilbaecher KN, Guise TA, McCauley LK. Cancer to bone: a fatal attraction. Nat Rev Cancer. 2011;11(6):411-425.

23. Marino S, Roodman GD. Multiple Myeloma and Bone: The Fatal Interaction. Cold Spring Harb Perspect Med. 2018;8(8):a031286.

24. Kawai T, et al. B and T lymphocytes are the primary sources of RANKL in the bone resorptive lesion of periodontal disease. Am J Pathol. 2006;169(3):987-998.

25. Nosaka K, Miyamoto T, Sakai T, Mitsuya H, Suda T, Matsuoka M. Mechanism of hypercalcemia in adult T-cell leukemia: overexpression of receptor activator of nuclear factor kappaB ligand on adult T-cell leukemia cells. Blood. 2002;99(2):634-640.

26. Shu ST, Martin CK, Thudi NK, Dirksen WP, Rosol TJ. Osteolytic bone resorption in adult T-cell leukemia/lymphoma. Leuk Lymphoma. 2010;51(4):702-714.

27. Nadella MV, et al. Expression of parathyroid hormone-related protein during immortalization of human peripheral blood mononuclear cells by HTLV-1: implications for transformation. Retrovirology. 2008;5:46.

28. Niewiesk S. Animals Models of Human T Cell Leukemia Virus Type I Leukemogenesis. ILAR J. 2016;57(1):3-11.

29. Walsh NC, et al. Humanized Mouse Models of Clinical Disease. Annu Rev Pathol. 2017;12:187-215.

30. Villaudy J, et al. HTLV-1 propels thymic human T cell development in "human immune system" Rag2 ${ }^{-/-}$gamma ${ }^{-/-}$mice. $P L o S$ Pathog. 2011;7(9):e1002231.

31. Huey DD, Niewiesk S. Production of Humanized Mice through Stem Cell Transfer. Curr Protoc Mouse Biol. 2018;8(1):17-27. 
32. Huey DD, et al. Role of Wild-type and Recombinant Human T-cell Leukemia Viruses in Lymphoproliferative Disease in Humanized NSG Mice. Comp Med. 2018;68(1):4-14.

33. Tezuka K, et al. An animal model of adult T-cell leukemia: humanized mice with HTLV-1-specific immunity. Blood. 2014;123(3):346-355.

34. Yamagishi M, et al. Polycomb-mediated loss of miR-31 activates NIK-dependent NF- $\mathrm{kB}$ pathway in adult T cell leukemia and other cancers. Cancer Cell. 2012;21(1):121-135.

35. Kwon H, et al. Lethal cutaneous disease in transgenic mice conditionally expressing type I human T cell leukemia virus Tax. J Biol Chem. 2005;280(42):35713-35722.

36. Yasuma K, et al. HTLV-1 bZIP Factor Impairs Anti-viral Immunity by Inducing Co-inhibitory Molecule, T Cell Immunoglobulin and ITIM Domain (TIGIT). PLoS Pathog. 2016;12(1):e1005372.

37. Bishop KA, Coy HM, Nerenz RD, Meyer MB, Pike JW. Mouse Rankl expression is regulated in T cells by c-Fos through a cluster of distal regulatory enhancers designated the T cell control region. J Biol Chem. 2011;286(23):20880-20891.

38. Bishop KA, Wang X, Coy HM, Meyer MB, Gumperz JE, Pike JW. Transcriptional regulation of the human TNFSF11 gene in T cells via a cell type-selective set of distal enhancers. J Cell Biochem. 2015;116(2):320-330.

39. Matsumoto J, Ohshima T, Isono O, Shimotohno K. HTLV-1 HBZ suppresses AP-1 activity by impairing both the DNA-binding ability and the stability of c-Jun protein. 2005;24(6):1001-1010.

40. Kostenuik PJ, et al. Denosumab, a fully human monoclonal antibody to RANKL, inhibits bone resorption and increases BMD in knock-in mice that express chimeric (murine/human) RANKL. J Bone Miner Res. 2009;24(2):182-195.

41. Lacey DL, et al. Bench to bedside: elucidation of the OPG-RANK-RANKL pathway and the development of denosumab. Nat Rev Drug Discov. 2012;11(5):401-419.

42. Walsh NC, et al. Activated human T cells express alternative mRNA transcripts encoding a secreted form of RANKL. Genes Immun. 2013;14(5):336-345.

43. Polakowski N, Gregory H, Mesnard JM, Lemasson I. Expression of a protein involved in bone resorption, Dkk1, is activated by HTLV-1 bZIP factor through its activation domain. Retrovirology. 2010;7:61.

44. Bellon M, et al. Adult T-cell leukemia cells overexpress Wnt5a and promote osteoclast differentiation. Blood. 2013;121(25):5045-5054.

45. Miranda-Carús ME, et al. Peripheral blood T lymphocytes from patients with early rheumatoid arthritis express RANKL and interleukin-15 on the cell surface and promote osteoclastogenesis in autologous monocytes. Arthritis Rheum. 2006;54(4):1151-1164.

46. Manz MG. Human-hemato-lymphoid-system mice: opportunities and challenges. Immunity. 2007;26(5):537-541.

47. Willinger T, Rongvaux A, Strowig T, Manz MG, Flavell RA. Improving human hemato-lymphoid-system mice by cytokine knock-in gene replacement. Trends Immunol. 2011;32(7):321-327.

48. Li Y, et al. Induction of functional human macrophages from bone marrow promonocytes by M-CSF in humanized mice. $J$ Immunol. 2013;191(6):3192-3199.

49. Melkus MW, et al. Humanized mice mount specific adaptive and innate immune responses to EBV and TSST-1. Nat Med. 2006;12(11):1316-1322.

50. Lan P, Tonomura N, Shimizu A, Wang S, Yang YG. Reconstitution of a functional human immune system in immunodeficient mice through combined human fetal thymus/liver and CD34+ cell transplantation. Blood. 2006;108(2):487-492.

51. Koralnik IJ, Lemp JF, Gallo RC, Franchini G. In vitro infection of human macrophages by human T-cell leukemia/lymphotropic virus type I (HTLV-I). AIDS Res Hum Retroviruses. 1992;8(11):1845-1849.

52. Rodrigues ES, et al. HTLV-1 infects human mesenchymal stromal cell in vitro and modifies their phenotypic characteristics. Virology. 2014;449:190-199.

53. Raynaud-Messina B, et al. Bone degradation machinery of osteoclasts: An HIV-1 target that contributes to bone loss. Proc Natl Acad Sci USA. 2018;115(11):E2556-E2565.

54. Rizzoli R, Yasothan U, Kirkpatrick P. Denosumab. Nat Rev Drug Discov. 2010;9(8):591-592.

55. Anderson K, et al. Role of Bone-Modifying Agents in Multiple Myeloma: American Society of Clinical Oncology Clinical Practice Guideline Update. J Clin Oncol. 2018;36(8):812-818.

56. Kondo A, et al. A model of in vivo cell proliferation of adult T-cell leukemia. Blood. 1993;82(8):2501-2509.

57. Masaki A, et al. Autologous Tax-specific CTL therapy in a primary adult T cell leukemia/lymphoma cell-bearing NOD/Shiscid, IL-2R $\gamma$ null mouse model. J Immunol. 2013;191(1):135-144.

58. Hiraragi $\mathrm{H}$, et al. Human T-lymphotropic virus type 1 mitochondrion-localizing protein p13(II) is required for viral infectivity in vivo. J Virol. 2006;80(7):3469-3476.

59. Su X, et al. The ADP receptor P2RY12 regulates osteoclast function and pathologic bone remodeling. J Clin Invest. 2012;122(10):3579-3592.

60. Sanjana NE, Shalem O, Zhang F. Improved vectors and genome-wide libraries for CRISPR screening. Nat Methods. 2014;11(8):783-784

61. Heller E, et al. Hedgehog signaling inhibition blocks growth of resistant tumors through effects on tumor microenvironment. Cancer Res. 2012;72(4):897-907.

62. Brunetto GS, et al. Digital droplet PCR (ddPCR) for the precise quantification of human T-lymphotropic virus 1 proviral loads in peripheral blood and cerebrospinal fluid of HAM/TSP patients and identification of viral mutations. J Neurovirol. 2014;20(4):341-351.

63. Fujikawa D, et al. Polycomb-dependent epigenetic landscape in adult T-cell leukemia. Blood. 2016;127(14):1790-1802.

64. Clough E, Barrett T. The Gene Expression Omnibus Database. Methods Mol Biol. 2016;1418:93-110.

65. Zhang Y, et al. Model-based analysis of ChIP-Seq (MACS). Genome Biol. 2008;9(9):R137.

66. Langmead B, Trapnell C, Pop M, Salzberg SL. Ultrafast and memory-efficient alignment of short DNA sequences to the human genome. Genome Biol. 2009;10(3):R25.

67. Kent WJ, et al. The human genome browser at UCSC. Genome Res. 2002;12(6):996-1006 